\title{
Adaptations of data mining methodologies: A systematic literature review
}

\author{
Veronika Plotnikova ${ }^{\text {Corresp., }}{ }^{1}$, Marlon Dumas $^{1}$, Fredrik Milani $^{1}$ \\ ${ }^{1}$ Institute of Computer Science, University of Tartu, Tartu, Estonia \\ Corresponding Author: Veronika Plotnikova \\ Email address: veronika.plotnikova@ut.ee
}

The use of end-to-end data mining methodologies such as CRISP-DM, KDD process, and SEMMA, has grown substantially over the past decade. However, little is known as to how these methodologies are used in practice. In particular, the question of whether data mining methodologies are used 'as-is' or adapted for specific purposes, has not been thoroughly investigated. This paper addresses this gap via a systematic literature review focused on the context in which data mining methodologies are used and the adaptations they undergo. The literature review covers 207 peer-reviewed and 'grey' publications. We find that data mining methodologies are primarily applied 'as-is'. At the same time, we also identify various adaptations of data mining methodologies and we note that their number is growing rapidly. The dominant adaptations pattern is related to methodology adjustments at a granular level (modifications) followed by extensions of existing methodologies with additional elements. Further, we identify two recurrent purposes for adaptation: (1) adaptations to handle Big Data technologies, tools and environments (technological adaptations); and (2) adaptations for context-awareness and for integrating data mining solutions into business processes and IT systems (organizational adaptations). The study suggests that standard data mining methodologies do not pay sufficient attention to deployment issues, which play a prominent role when turning data mining models into software products that are integrated into the IT architectures and business processes of organizations. We conclude that refinements of existing methodologies aimed at combining data, technological, and organizational aspects, could help to mitigate these gaps. 


\title{
Adaptations of Data Mining Methodologies: A Systematic Literature Review
}

3 Veronika Plotnikova, Marlon Dumas, and Fredrik P. Milani

\author{
University of Tartu, Institute of Computer Science, Narva mnt 18, 51009 Tartu, Estonia \\ Corresponding author: \\ Veronika Plotnikova \\ Email address: veronika.plotnikova@ut.ee
}

\section{ABSTRACT}

\begin{abstract}
The use of end-to-end data mining methodologies such as CRISP-DM, KDD process, and SEMMA, has grown substantially over the past decade. However, little is known as to how these methodologies are used in practice. In particular, the question of whether data mining methodologies are used 'as-is' or adapted for specific purposes, has not been thoroughly investigated. This paper addresses this gap via a systematic literature review focused on the context in which data mining methodologies are used and the adaptations they undergo. The literature review covers 207 peer-reviewed and 'grey' publications. We find that data mining methodologies are primarily applied 'as-is'. At the same time, we also identify various adaptations of data mining methodologies and we note that their number is growing rapidly. The dominant adaptations pattern is related to methodology adjustments at a granular level (modifications) followed by extensions of existing methodologies with additional elements. Further, we identify two recurrent purposes for adaptation: (1) adaptations to handle Big Data technologies, tools and environments (technological adaptations); and (2) adaptations for context-awareness and for integrating data mining solutions into business processes and IT systems (organizational adaptations). The study suggests that standard data mining methodologies do not pay sufficient attention to deployment issues, which play a prominent role when turning data mining models into software products that are integrated into the IT architectures and business processes of organizations. We conclude that refinements of existing methodologies aimed at combining data, technological, and organizational aspects, could help to mitigate these gaps.
\end{abstract}

Keywords: Data Mining, Data Mining Methodologies, CRISP-DM, Literature Review

\section{INTRODUCTION}

The availability of Big Data has stimulated widespread adoption of data mining and data analytics in research and in business settings (Columbus, 2017). Over the years certain number of data mining methodologies have been proposed too, and they are being used extensively in practice and in research. However, little is known about what and how data mining methodologies are applied, and it has not been neither widely researched nor discussed. Further, there is no consolidated view on what constitutes quality of methodological process in data mining and data analytics, how data mining and data analytics are applied/used in organization settings context, and how application practices relate to each other. That motivates the need for comprehensive survey in the field.

There have been surveys or quasi-surveys and summaries conducted in related fields. Notably, there have been two systematic systematic literature reviews; Systematic Literature Review, hereinafter, SLR is the most suitable and widely used research method for identifying, evaluating and interpreting research of particular research question, topic or phenomenon (Kitchenham et al., 2015). These reviews concerned Big Data Analytics, but not general purpose data mining methodologies. Adrian et al. (2004) executed SLR with respect to implementation of Big Data Analytics (BDA), specifically, capability components necessary for BDA value discovery and realization. The authors identified BDA implementation studies, determined their main focus areas, and discussed in detail BDA applications and capability components. Saltz and Shamshurin (2016) have published SLR paper on Big Data Team Process Methodologies. Authors have identified lack of standard in regards to how Big Data projects are executed, highlighted growing research in this area and potential benefits of such process standard. Additionally, authors

Peer] Comput. Sci. reviewing PDF | (CS-2019:06:38457:2:0:NEW 19 Feb 2020) 
synthesize and produced list of 33 most important success factors for executing Big Data activities. Finally, there are studies that surveyed data mining techniques and applications across domains, yet, they focus on data mining process artefacts and outcomes (eg. Madni et al. (2017), Liao et al. (2012)), but not on end-to-end process methodology.

There have been number of surveys conducted in domain-specific settings such as hospitality, accounting, education, manufacturing, and banking fields. Mariani et al. (2018) focused on $\mathrm{BI}^{1}$ and Big Data SLR in the hospitality and tourism environment context. Amani and Fadlalla (2017) explored application of data mining methods in accounting while Romero and Ventura (2013) investigated educational data mining. Similarly, Hassani et al. (2018) addressed data mining application case studies in banking and explored them by three dimensions - topics, applied techniques and software. All studies were performed by the means of systematic literature reviews. Lastly, Bi and Cochran (2014) have undertaken standard literature review of Big Data Analytics and its applications in manufacturing.

Apart from domain-specific studies, there have been very few general purpose surveys with comprehensive overview of existing data mining methodologies, classifying and contextualizing them. Valuable synthesis was presented by Kurgan and Musílek (2006) as comparative study of the state-of-the art of data mining methodologies. The study was not SLR, and focused on comprehensive comparison of phases, processes, activities of data mining methodologies; application aspect was summarized briefly as application statistics by industries and citations. Three more comparative, non-SLR studies were undertaken by Marban et al. (2009), Mariscal et al. (2010), and the most recent and closest one by Martínez-Plumed et al. (2017). They followed the same pattern with systematization of existing data mining frameworks based on comparative analysis. There, the purpose and context of consolidation was even more practical to support derivation and proposal of the new artefact, i.e. novel data mining methodology. Majority of the given general type surveys in the field are more than a decade old, and have natural limitations due to being: (1) non-SLR studies, and (2) so far restricted to comparing methodologies in terms of phases, activities, and other elements.

The key common characteristic behind all the given studies is that data mining methodologies are treated as normative and standardized ('one-size-fits-all') processes. A complementary perspective, not considered in the above studies, is that data mining methodologies are not normative standardized processes, but instead, they are frameworks that need to be specialized to different industry domains, organizational contexts, and business objectives. In the last few years, a number of extensions and adaptations of data mining methodologies have emerged, which suggest that existing methodologies are not sufficient to cover the needs of all application domains. In particular, extensions of data mining methodologies have been proposed in the medical domain (Niaksu, 2015), educational domain (Tavares et al., 2017), the industrial engineering domain (Huber et al., 2019), (Solarte, 2002), and software engineering (Marbán et al., 2007), (Marbán et al., 2009). However, little attention has been given to studying how data mining methodologies are applied and used in industry settings, so far only nonscientific practitioners' surveys provide such evidence.

Given this research gap, the central objective of the paper is to investigate how data mining methodologies are applied by researchers and practitioners, both in their generic (standardized) form and in specialized settings. This is achieved by

investigating if data mining methodologies are applied 'as-is' or adapted, and for what purposes such adaptations are implemented.

Guided by Systematic Literature Review method, initially we identified a corpus of primary studies covering both peer-reviewed and 'grey' literature from 1997 to 2018. An analysis of these studies led us to a taxonomy of uses of data mining methodologies, focusing on the distinction between 'as is' usage versus various types of methodology adaptations. By analyzing different types of methodology adaptations, the paper identifies potential gaps in standard data mining methodologies both at the technological and at the organizational levels.

The rest of the paper is organized as follows. Section Background provides an overview of key concepts of data mining and associated methodologies. Next, Section Research Design describes the research methodology. Section Findings and Discussion presents the study results and their associated interpretation. Finally, threats to validity are addressed in Threats to Validity while Conclusion summarizes the findings and outlines directions for future work.

${ }^{1}$ Business Intelligence 


\section{BACKGROUND}

The section introduces main data mining concepts, provides overview of existing data mining methodologies, and their evolution.

Data mining is defined as a set of rules, processes, algorithms that are designed to generate actionable insights, extract patterns, and identify relationships from large data sets (Morabito, 2016). Data mining incorporates automated data extraction, processing, and modeling by means of a range of methods and techniques. In contrast, data analytics refers to techniques used to analyze and acquire intelligence from data (including 'big data') (Gandomi and Haider, 2015) and is positioned as a broader field, encompassing a wider spectrum of methods that includes both statistical and data mining (Chen et al., 2012). A number of algorithms has been developed in statistics, machine learning, and artificial intelligence domains to support and enable data mining. While statistical approaches precedes them, they inherently come with limitations, the most known being rigid data distribution conditions. Machine learning techniques gained popularity as they impose less restrictions while deriving understandable patterns from data (Bose and Mahapatra, 2001).

Data mining projects commonly follow a structured process or methodologyas exemplified by Mariscal et al. (2010), Marban et al. (2009). A data mining methodology specifies tasks, inputs, outputs, and provides guidelines and instructions on how the tasks are to be executed. (Mariscal et al., 2010). Thus, data mining methodology provides a set of guidelines for executing a set of tasks to achieve the objectives of a data mining project. (Mariscal et al., 2010).

The foundations of structured data mining methodologies were first proposed by Fayyad et al. (1996a), Fayyad et al. (1996b), Fayyad et al. (1996c), and were initially related to Knowledge Discovery in Databases (KDD). KDD presents a conceptual process model of computational theories and tools that support information extraction (knowledge) with data (Fayyad et al., 1996a). In KDD, the overall approach to knowledge discovery includes data mining as a specific step. As such, KDD, with its nine main steps (exhibited in Figure 1 below), has the advantage of considering data storage and access, algorithm scaling, interpretation and visualization of results, and human computer interaction (Fayyad et al., 1996a), (Fayyad et al., 1996c). Introduction of KDD also formalized clearer distinction between data mining and data analytics, as for example formulated in Tsai et al. (2015): '...by the data analytics, we mean the whole KDD process, while by the data analysis, we mean the part of data analytics that is aimed at finding the hidden information in the data, such as data mining'.

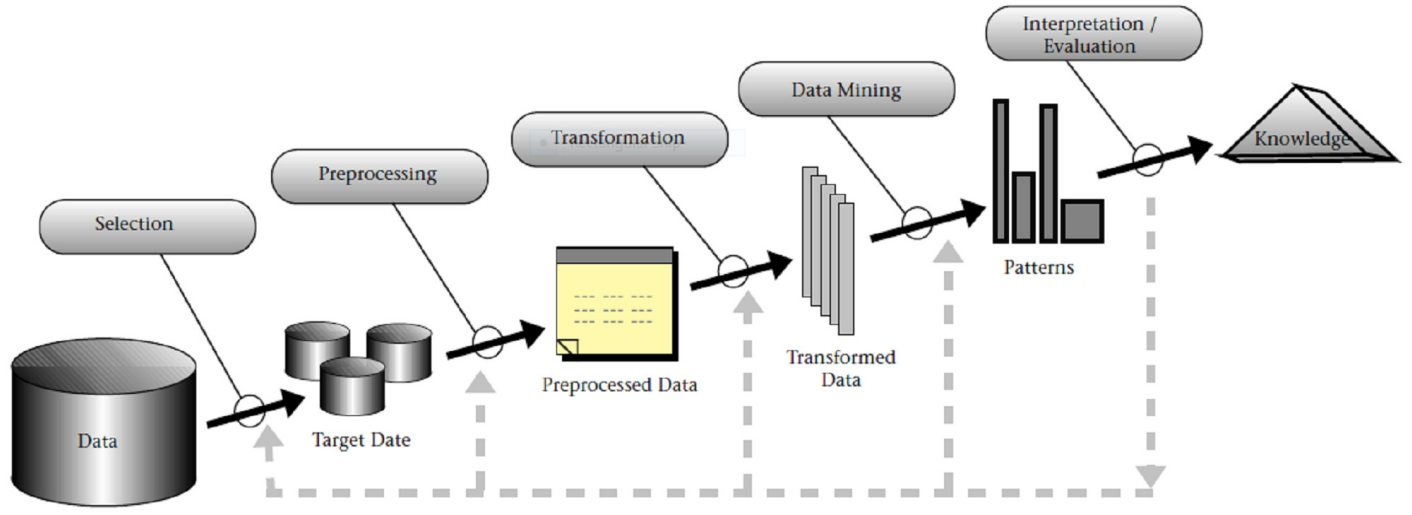

Figure 1. An overview of the steps composing the KDD process, as presented in Fayyad et al. (1996a), Fayyad et al. (1996c)

The main steps of KDD are as follows:

- Step 1 - Learning application domain: In the first step, it is needed to develop an understanding of the application domain and relevant prior knowledge followed by identifying the goal of the KDD process from the customer's viewpoint. 
- Step 2 - Data set creation: Second step involves selecting a data set, focusing on a subset of variables or data samples on which discovery is to be performed.

- Step 3 - Data cleaning and processing: In the third step, basic operations to remove noise or outliers are performed. Collection of necessary information to model or account for noise, deciding on strategies for handling missing data fields, and accounting for data types, schema, and mapping of missing and unknown values are also considered.

- Step 4 - Data reduction and projection: Here, the work of finding useful features to represent the data, depending on the goal of the task, application of transformation methods to find optimal features set for the data is conducted.

- Step 5 - Choosing the function of data mining: In the fifth step, the target outcome (e.g., summarization, classification, regression, clustering) are defined.

- Step 6 - Choosing data mining algorithm: Sixth step concerns selecting method(s) to search for patterns in the data, deciding which models and parameters are appropriate and matching a particular data mining method with the overall criteria of the KDD process.

- Step 7 - Data mining: In the seventh step, the work of mining the data i.e., searching for patterns of interest in a particular representational form or a set of such representations: classification rules or trees, regression, clustering is conducted.

- Step 8 - Interpretation: In this step, the redundant and irrelevant patterns are filtered out, relevant patterns are interpreted and visualized in such way as to make the result understandable to the users.

- Step 9 - Using discovered knowledge: In the last step, the results are incorporated with the performance system, documented and reported to stakeholders, and used as basis for decisions.

The KDD process became dominant in industrial and academic domains (Kurgan and Musílek, 2006), (Marban et al., 2009). Also, as timeline-based evolution of data mining methodologies and process models shows (Figure 2 below), the original KDD data mining model served as basis for other methodologies and process models, which addressed various gaps and deficiencies of original KDD process. These approaches extended the initial KDD framework, yet, extension degree has varied ranging from process restructuring to complete change in focus. For example, Brachman and Anand (1996) and further Gertosio and Dussauchoy (2004) (in a form of case study) introduced practical adjustments to the process based on iterative nature of process as well as interactivity. The complete KDD process in their view was enhanced with supplementary tasks and the focus was changed to user's point of view (human-centered approach), highlighting decisions that need to be made by the user in the course of data mining process. In contrast, Cabena et al. (1997) proposed different number of steps emphasizing and detailing data processing and discovery tasks. Similarly, in a series of works Anand and Büchner (1998), Anand et al. (1998), Buchner et al. (1999) presented additional data mining process steps by concentrating on adaptation of data mining process to practical settings. They focused on cross-sales (entire life-cycles of online customer), with further incorporation of internet data discovery process (web-based mining). Further, Two Crows data mining process model is consultancy originated framework that has defined the steps differently, but is still close to original KDD. Finally, SEMMA (Sample, Explore, Modify, Model and Assess) based on KDD, was developed by SAS institute in 2005 (SAS Institute, 2017). It is defined as a logical organization of the functional toolset of SAS Enterprise Miner for carrying out the core tasks of data mining. Compared to $\mathrm{KDD}$, this is vendor-specific process model which limits its application in different environments. Also, it skips two steps of original KDD process ('Learning Application Domain' and 'Using of Discovered Knowledge') which are regarded as essential for success of data mining project (Mariscal et al., 2010). In terms of adoption, new KDD-based proposals received limited attention across academia and industry (Kurgan and Musílek, 2006), (Marban et al., 2009). Subsequently, most of these methodologies converged into the CRISP-DM methodology.

Additionally, there have only been two non-KDD based approaches proposed alongside extensions to KDD. The first one is 5A's approach presented by de Pisón Ascacíbar (2003) and used by SPSS vendor. The key contribution of this approach has been related to adding 'Automate' step while disadvantage was associated with omitting 'Data Understanding' step. The second approach was 6-Sigma which is industry 


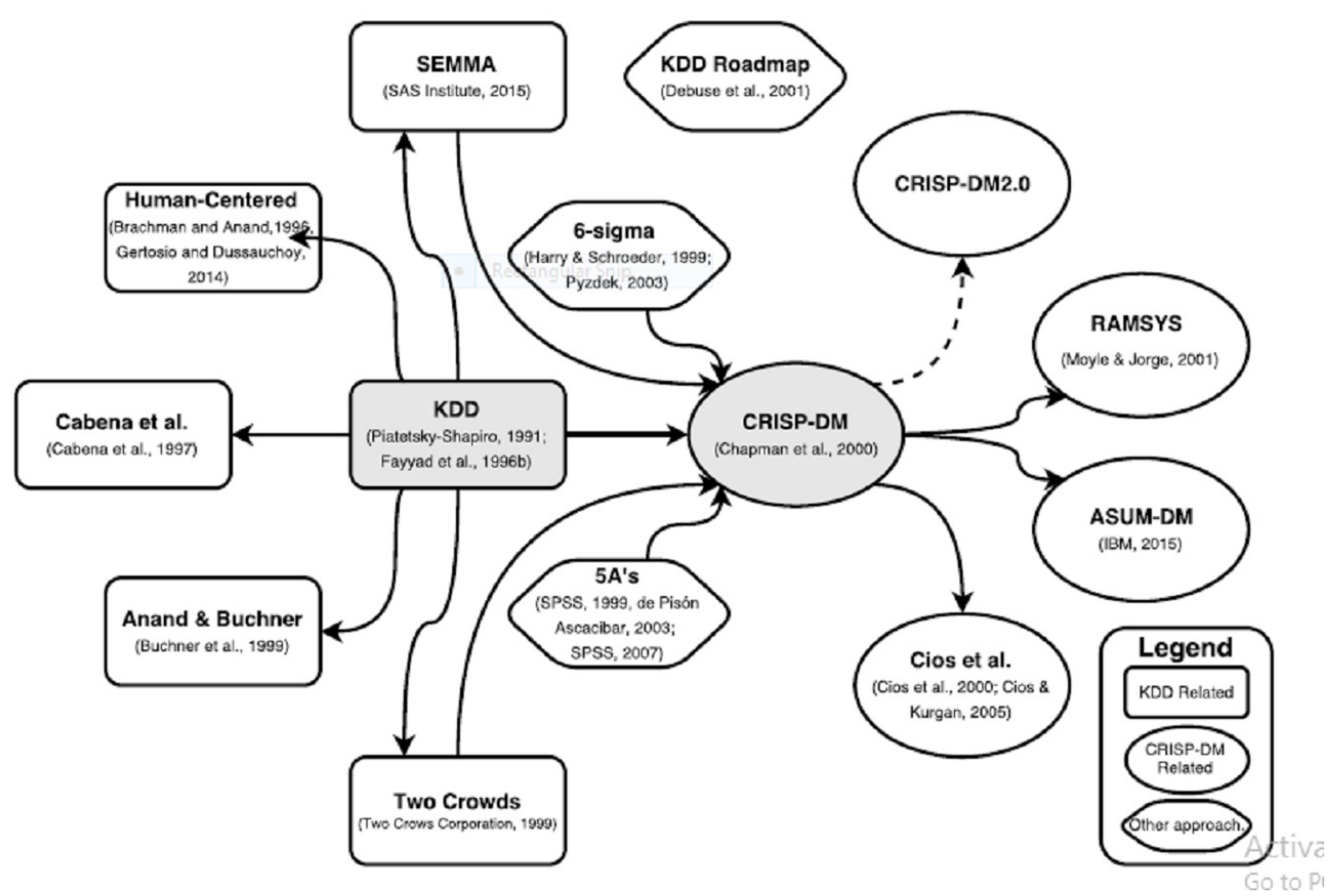

Figure 2. Evolution of data mining process and methodologies, as presented in Martínez-Plumed et al. (2017)

originated method to improve quality and customer's satisfaction (Pyzdek and Keller, 2003). It has been successfully applied to data mining projects in conjunction with DMAIC performance improvement model (Define, Measure, Analyze, Improve, Control).

In 2000, as response to common issues and needs (Marban et al., 2009), an industry-driven methodology called Cross-Industry Standard Process for Data Mining (CRISP-DM) was introduced as an alternative to KDD. It also consolidated original KDD model and its various extensions. While CRISP-DM builds upon KDD, it consists of six phases that are executed in iterations (Marban et al., 2009). The iterative executions of CRISP-DM stands as the most distinguishing feature compared to initial KDD that assumes a sequential execution of its steps. CRISP-DM, much like KDD, aims at providing practitioners with guidelines to perform data mining on large data sets. However,CRISP-DM with its six main steps with a total of 24 tasks and outputs, is more refined as compared to KDD. The main steps of CRIPS-DM, as depicted in Figure 3 below are as follows:

- Phase 1- Business understanding: The focus of the first step is to gain an understanding of - of the project objectives and requirements from a business perspective followed by converting these into data mining problem definitions. Presentation of a preliminary plan to achieve the objectives are also included in this first step.

- Phase 2 - Data understanding: This step begins with an initial data collection and proceeds with activities in order to get familiar with the data, identify data quality issues, discover first insights into the data, and potentially detect and form hypotheses.

- Phase 3 - Data preparation: The third step covers activities required to construct the final data set from the initial raw data. Data preparation tasks are performed repeatedly.

- Phase 4 -Modeling phase: In this step, various modeling techniques are selected and applied followed by calibrating their parameters. Typically, several techniques are used for the same data mining problem.

- Phase 5 - Evaluation of the model(s): The fifth step begins with the quality perspective and then, 


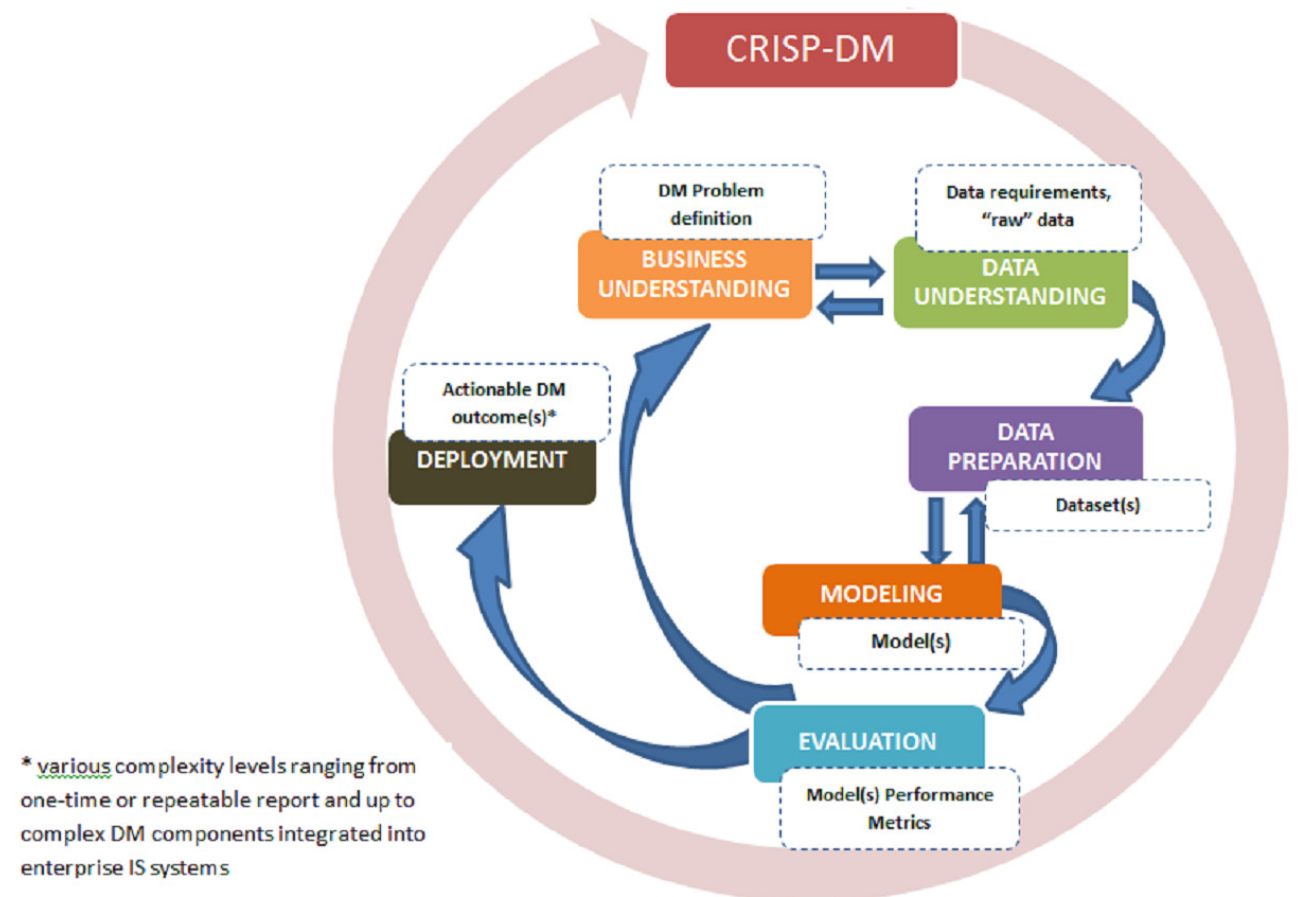

Figure 3. CRISP-DM phases and key outputs (adapted from (Chapman et al., 2000))

before proceeding to final model deployment, ascertains that the model(s) achieves the business objectives. At the end of this phase, a decision should be reached on how to use data mining results.

- Phase 6 - Deployment phase: In the final step, the models are deployed to enable end-customers to use the data as basis for decisions, or support in the business process. Even if the purpose of the model is to increase knowledge of the data, the knowledge gained will need to be organized, presented, distributed in a way that the end-user can use it. Depending on the requirements, the deployment phase can be as simple as generating a report or as complex as implementing a repeatable data mining process.

The development of CRISP-DM was led by industry consortium. It is designed to be domain-agnostic (Mariscal et al., 2010) and as such, is now widely used by industry and research communities (Marban et al., 2009). These distinctive characteristics have made CRISP-DM to be considered as 'de-facto' standard of data mining methodology and as a reference framework to which other methodologies are benchmarked (Mariscal et al., 2010).

Similarly to KDD, a number of refinements and extensions of the CRISP-DM methodology have been proposed with the two main directions - extensions of the process model itself and adaptations, merger with the process models and methodologies in other domains. Extensions direction of process models could be exemplified by Cios and Kurgan (2005) who have proposed integrated DMKD (Data Mining and Knowledge Discovery) process model. It contains several explicit feedback mechanisms, modification of the last step to incorporate discovered knowledge and insights application as well as relies on technologies for results deployment. In the same vein, Moyle and Jorge (2001), Blockeel and Moyle (2002) proposed Rapid Collaborative Data Mining System (RAMSYS) framework - this is both data mining methodology and system for remote collaborative data mining projects. The RAMSYS attempted to achieve the combination of a problem solving methodology, knowledge sharing, and ease of communication. It intended to allow the collaborative work of remotely placed data miners in a disciplined manner as regards information flow while allowing the free flow of ideas for problem solving (Moyle and Jorge, 2001). CRISP-DM modifications and integrations with other specific domains were proposed in Industrial Engineering (Data Mining for Industrial Engineering by Solarte (2002)), and Software Engineering by Marbán et al. (2007), Marbán et al. (2009). Both approaches enhanced CRISP-DM and 


\begin{tabular}{|c|c|c|c|c|}
\hline Name & Origin & Basis & Key concept & Year \\
\hline $\begin{array}{l}\text { Human- } \\
\text { Centered }\end{array}$ & Academy & KDD & $\begin{array}{l}\text { Iterative process and interactivity } \\
\text { (user's point of view and needed } \\
\text { decisions) }\end{array}$ & $\begin{array}{l}1996, \\
2004\end{array}$ \\
\hline Cabena et al & Academy & KDD & $\begin{array}{l}\text { Focus on data processing and discovery } \\
\text { tasks }\end{array}$ & 1997 \\
\hline $\begin{array}{l}\text { Anand and } \\
\text { Buchner }\end{array}$ & Academy & KDD & $\begin{array}{l}\text { Supplementary steps and integration of } \\
\text { web-mining }\end{array}$ & $\begin{array}{l}1998, \\
1999\end{array}$ \\
\hline Two Crows & Industry & KDD & Modified definitions of steps & 1998 \\
\hline SEMMA & Industry & KDD & $\begin{array}{l}\text { Tool-specific (SAS Institute), elimina- } \\
\text { tion of some steps }\end{array}$ & 2005 \\
\hline $5 A^{\prime} s$ & Industry & Independent & Supplementary steps & 2003 \\
\hline 6 Sigmas & Industry & Independent & $\begin{array}{l}6 \text { Sigma quality improvement paradigm } \\
\text { in conjunction with DMAIC perfor- } \\
\text { mance improvement model }\end{array}$ & 2003 \\
\hline CRISP-DM & $\begin{array}{l}\text { Joint industry } \\
\text { and academy }\end{array}$ & KDD & $\begin{array}{l}\text { Iterative execution of steps, significant } \\
\text { refinements to tasks and outputs }\end{array}$ & 2000 \\
\hline Cios et al & Academy & Crisp-DM & $\begin{array}{l}\text { Integration of data mining and knowl- } \\
\text { edge discovery, feedback mechanisms, } \\
\text { usage of received insights supported by } \\
\text { technologies }\end{array}$ & 2005 \\
\hline RAMSYS & Academy & Crisp-DM & $\begin{array}{l}\text { Integration of collaborative work as- } \\
\text { pects }\end{array}$ & $\begin{array}{l}2001- \\
2002\end{array}$ \\
\hline DMIE & Academy & Crisp-DM & $\begin{array}{l}\text { Integration and adaptation to Industrial } \\
\text { Engineering domain }\end{array}$ & 2001 \\
\hline Marban & Academy & Crisp-DM & $\begin{array}{l}\text { Integration and adaptation to Software } \\
\text { Engineering domain }\end{array}$ & 2007 \\
\hline $\begin{array}{l}\text { KDD } \\
\text { roadmap }\end{array}$ & $\begin{array}{l}\text { Joint industry } \\
\text { and academy }\end{array}$ & Independent & Tool-specific, resourcing task & 2001 \\
\hline ASUM & Industry & Crisp-DM & $\begin{array}{l}\text { Tool-specific, combination of traditional } \\
\text { Crisp-DM and agile implementation ap- } \\
\text { proach }\end{array}$ & 2015 \\
\hline
\end{tabular}

Table 1. Key aspects of existing Data Mining process models and methodologies

contributed with additional phases, activities and tasks typical for engineering processes, addressing on-going support (Solarte, 2002), as well as project management, organizational and quality assurance tasks (Marbán et al., 2009).

Finally, limited number of attempts to create independent or semi-dependent data mining frameworks was undertaken after CRISP-DM creation. These efforts were driven by industry players and comprised KDD Roadmap by Debuse et al. (2001) for proprietary predictive toolkit (Lanner Group), and recent effort by IBM with Analytics Solutions Unified Method for Data Mining (ASUM-DM) in 2015 (IBM, $2016)^{2}$. Both frameworks contributed with additional tasks, eg. resourcing in KDD Roadmap, or hybrid approach assumed in ASUM, eg. combination of agile and traditional implementation principles.

The Table 1 above summarizes reviewed data mining process models and methodologies by their origin, basis and key concepts.

\section{RESEARCH DESIGN}

The main research objective of this paper is to study how data mining methodologies are applied by researchers and practitioners. To this end, we use systematic literature review (SLR) as scientific method for two reasons. Firstly, systematic review is based on trustworthy, rigorous, and auditable methodology. Secondly, SLR supports structured synthesis of existing evidence, identification of research gaps, and

${ }^{2}$ Available at ftp://ftp.software.ibm.com/software/data/sw-library/services/A.Sum.pdf 
provides framework to position new research activities (Kitchenham et al., 2015). For our SLR, we followed the guidelines proposed by Kitchenham et al. (2015). All SLR details have been documented in the separate, peer-reviewed SLR protocol (available at link ${ }^{3}$ ).

\section{Research Questions}

As suggested by Kitchenham et al. (2015), we have formulated research questions and motivate them as follows. In the preliminary phase of research we have discovered very limited number of studies investigating data mining methodologies application practices as such. Further, we have discovered number of surveys conducted in domain-specific settings, and very few general purpose surveys, but none of them considered application practices either. As contrasting trend, recent emergence of limited number of adaptation studies have clearly pinpointed the research gap existing in the area of application practices. Given this research gap, in-depth investigation of this phenomenon led us to ask 'How data mining methodologies are applied ('as-is' vs adapted) (RQ1)? Further, as we intended to investigate in depth universe of adaptations scenarios, this naturally led us to RQ2, 'How have existing data mining methodologies been adapted?'. Finally, if adaptions are made, we wish to explore what the associated reasons and purposes are, which in turn led us to RQ3, 'For what purposes are data mining methodologies adapted?'.

Thus, for this review, they are three research questions defined:

- Research Question 1: How data mining methodologies are applied ('as-is' versus adapted)? this question aims to identify data mining methodologies application and usage patterns and trends.

- Research Question 2: How have existing data mining methodologies been adapted? - this questions aims to identify and classify data mining methodologies adaptation patterns and scenarios.

- Research Question 3: For what purposes have existing data mining methodologies been adapted? - this question aims to identify, explain, classify and produce insights on what are the reasons and what benefits are achieved by adaptations of existing data mining methodologies. Specifically, what gaps do these adaptations seek to fill and what have been the benefits of these adaptations. Such systematic evidence and insights will be valuable input to potentially new, refined data mining methodology. Insights will be of interest to practitioners and researchers.

\section{Data Collection Strategy}

Our data collection and search strategy follow the guidelines proposed by Kitchenham et al. (2015). It defined the scope of the search, selection of literature and electronic databases, search terms and strings as well as screening procedures.

Primary Search The primary search aimed to identify an initial set of papers. To this end, the search strings were derived from the research objective and research questions. The term 'data mining' was the key term, but we also included 'data analytics' to be consistent with observed research practices. The terms 'methodology' and 'framework' were also included. Thus, the following search strings were developed and validated in accordance with the guidelines suggested by Kitchenham et al. (2015):

('data mining methodology') OR ('data mining framework') OR ('data analytics methodology') OR ('data analytics framework')

The search strings were applied to the indexed scientific databases Scopus, Web of Science (for 'peer-reviewed', academic literature) and to the non-indexed Google Scholar (for non-peer-reviewed, so-called "grey" literature). The decision to cover "grey" literature in this research was motivated as follows. As proposed in number of information systems and software engineering domain publications (eg. Garousi et al. (2019) - Neto et al. (2019)), SLR as stand-alone method may not provide sufficient insight into 'state of practice'. It was also identified (eg. in Garousi et al. (2016)) that 'grey' literature can give substantial benefits in certain areas of software engineering, in particular, when the topic of research is related to industrial and practical settings. Taking into consideration the research objectives, which is investigating data mining methodologies application practices, we have opted for inclusion of elements of Multivocal Literature Review (MLR) ${ }^{4}$ in our study. Also, (Kitchenham et al., 2015) recommends

\footnotetext{
${ }^{3}$ https://figshare.com/articles/Systematic-Literature-Review-Protocol/10315961

${ }^{4}$ Multivocal Literature Review (MLR) (as in Garousi et al. (2019) is a form of a SLR which includes the grey literature (e.g., blog posts, videos and white papers) in addition to the published (formal) literature (e.g., journal and conference papers).
} 


\footnotetext{
5 Including white papers, market reports, industry overviews and similar

${ }^{6}$ Excluded domains were Medicine, Biochemistry, Genetics and Molecular Biology, Environmental Science, Earth and Planetary Science, Physics and Astronomy, Energy and Material Science, Agricultural and Biological Science, Chemistry and Chemical Engineering, Pharmacology, Toxicology and Pharmaceuticals, Arts and Humanities, Neuroscience, Immunology and Microbiology, Health Professions and Nursing

including 'grey' literature to minimize publication bias as positive results and research outcomes are more likely to be published than negative ones. Following MLR practices, we also designed inclusion criteria for types of 'grey' literature reported below.

The selection of databases is motivated as follows. In case of peer-reviewed literature sources we concentrated to avoid potential omission bias. The latter is discussed in IS research (eg. (Levy and Ellis, 2006)) in case research is concentrated in limited disciplinary data sources. Thus, broad selection of data sources including multidisciplinary-oriented (Scopus, Web of Science, Wiley Online Library) and domain-oriented (ACM Digital Library, IEEE Xplorer Digital Library) scientific electronic databases was evaluated. Multidisciplinary databases have been selected due to wider domain coverage and it was validated and confirmed that they do include publications originating from domain-oriented databases, such as ACM and IEEE. From multi-disciplinary databases as such, Scopus was selected due to widest possible coverage (it is worlds largest database, covering app. 80\% of all international peer-reviewed journals) while Web of Science was selected due to its longer temporal range. Thus, both databases complement each other. The selected non-indexed database source for 'grey' literature is Google Scholar, as it is comprehensive source of both academic and 'grey' literature publications and referred as such extensively (eg. Garousi et al. (2019)-Neto et al. (2019)).

Further, Garousi et al. (2019) presented three-tier categorization framework for types of 'grey literature'. In our study we restricted ourselves to the 1 st tier 'grey' literature publications of the limited number of 'grey' literature producers. In particular, from the list of producers (Neto et al. (2019)) we have adopted and focused on government departments and agencies, non-profit economic, trade organizations ('thinktanks') and professional associations, academic and research institutions, businesses and corporations (consultancy companies and established private companies). The 1st tier 'grey' literature selected items include: (1) government, academic, and private sector consultancy reports ${ }^{5},(2)$ theses (not lower than Master level) and PhD Dissertations, (3) research reports, (4) working papers, (5) conference proceedings, preprints. With inclusion of the 1 st tier 'grey' literature criteria we mitigate quality assessment challenge especially relevant and reported for it (see for example Garousi et al. (2019)-Neto et al. (2019)).

Scope and Domains inclusion As recommended by (Kitchenham et al., 2015) it is necessary to initially define research scope. To clarify the scope, we defined what is not included and is out of scope of this research. The following aspects are not included in the scope of our study:

1. context of technology and infrastructure for data mining/data analytics tasks and projects.

2. granular methods application in data mining process itself or their application for data mining tasks, eg. constructing business queries or applying regression or neural networks modeling techniques to solve classification problems. Studies with granular methods are included in primary texts corpus as long as method application is part of overall methodological approach.

3. technological aspects in data mining eg. data engineering, dataflows and workflows.

4. traditional statistical methods not associated with data mining directly including statistical control methods.

Similarly to Budgen et al. (2006), Levy and Ellis (2006), initial piloting revealed that search engines retrieved literature available for all major scientific domains including ones outside authors' area of expertise (eg. medicine). Even though such studies could be retrieved, it would be impossible for us to analyze and correctly interpret literature published outside the possessed area of expertise. The adjustments toward search strategy were undertaken by retaining domains closely associated with Information Systems, Software Engineering research. Thus, for Scopus database the final set of inclusive domains was limited to nine and included Computer Science, Engineering, Mathematics, Business, Management and Accounting, Decision Science, Economics, Econometrics and Finance, and Multidisciplinary as well as Undefined studies. Excluded domains covered $11.5 \%$ or 106 out of 925 publications; it was confirmed in validation process that they primarily focused on specific case studies in fundamental sciences and medicine ${ }^{6}$. The 
included domains from Scopus database were mapped to Web of Science to ensure consistent approach across databases and the correctness of mapping was validated.

Screening criteria and procedures Based on the SLR practices (as in Kitchenham et al. (2015), Brereton et al. (2007)) and defined SLR scope, we designed multi-step screening procedures (quality and relevancy) with associated set of Screening Criteria and Scoring System. The purpose of relevancy screening is to find relevant primary studies in an unbiased way (Vanwersch et al., 2011). Quality screening, on the other hand, aims to assess primary relevant studies in terms of quality in unbiased way.

Screening Criteria consisted of two subsets - Exclusion Criteria applied for initial filtering and Relevance Criteria, also known as Inclusion Criteria.

Exclusion Criteria were initial threshold quality controls aiming at eliminating studies with limited or no scientific contribution. The exclusion criteria also address issues of understandability, accessability, and availability. The Exclusion Criteria were as follows:

1. Quality 1 - the publication item is not in English (understandability).

2. Quality 2 - publication item duplicates which can occur when:

- either the same document retrieved from two or all three databases.

- or different versions of the same publication are retrieved (i.e. the same study published in different sources) - based on best practices, decision rule is that the most recent paper is retained as well as the one with the highest score (Kofod-Petersen, 2014).

- if a publication is published both as conference proceeding and as journal article with the same name and same authors or as an extended version of conference paper, the latter is selected.

3. Quality 3 - length of the publication is less than 6 pages - short papers do not have the space to expand and discuss presented ideas in sufficient depth to examine for us.

4. Quality 4 - the paper is not accessible in full length online through the university subscription of databases and via Google Scholar - not full availability prevents us from assessing and analyzing the text.

The initially retrieved list of papers was filtered based on Exclusion Criteria. Only papers that passed all criteria were retained in the final studies corpus. Mapping of criteria towards screening steps is exhibited in Figure 4.

Relevance Criteria were designed to identify relevant publications and are presented in Table 2 below while mapping to respective process steps is presented in Figure 4 . These criteria were applied iteratively.

As a final SLR step, the full texts quality assessment was performed with constructed Scoring Metrics (in line with Kitchenham and Charters (2007)). It is presented in the Table 3 below.

\section{Data Extraction and Screening Process}

The conducted data extraction and screening process is presented in Figure 4. In Step 1 initial publications list were retrieved from pre-defined databases - Scopus, Web of Science, Google Scholar. The lists were merged and duplicates eliminated in Step 2. Afterwards, texts being less than 6 pages were excluded (Step 3). Steps 1-3 were guided by Exclusion Criteria. In the next stage (Step 4), publications were screened by Title based on pre-defined Relevance Criteria. The ones which passed were evaluated by their availability (Step 5). As long as study was available, it was evaluated again by the same pre-defined Relevance Criteria applied to Abstract, Conclusion and if necessary Introduction (Step 6). The ones which passed this threshold formed primary publications corpus extracted from databases in full. These primary texts were evaluated again based on full text (Step 7) applying Relevance Criteria first and then Scoring Metrics. 


\begin{tabular}{l|l|l}
$\begin{array}{l}\text { Relevance } \\
\text { Criteria }\end{array}$ & Criteria Definition & Criteria Justification \\
\hline Relevance 1 & $\begin{array}{l}\text { Is the study about data min- } \\
\text { ing or data analytics ap- } \\
\text { proach and is within desig- } \\
\text { nated list of domains? }\end{array}$ & $\begin{array}{l}\text { Exclude studies conducted outside the des- } \\
\text { ignated domain list. Exclude studies not } \\
\text { directly describing and/or discussing data } \\
\text { mining and data analytics }\end{array}$ \\
Relevance 2 & $\begin{array}{l}\text { Is the study introduc- } \\
\text { ing/describing data mining } \\
\text { or data analytics methodol- } \\
\text { ogy/framework or modifying } \\
\text { existing approaches? }\end{array}$ & $\begin{array}{l}\text { Exclude texts considering only specific, } \\
\text { granular data mining and data analytics } \\
\text { techniques, methods or traditional statisti- } \\
\text { cal methods. Exclude publications focus- } \\
\text { ing on specific, granular data mining and } \\
\text { data analytics process/sub-process aspects. } \\
\text { Exclude texts where description and dis- } \\
\text { cussion of data mining methodologies or } \\
\text { frameworks is manifestly missing }\end{array}$ \\
\hline
\end{tabular}

Table 2. Relevance Criteria mapping to screening process steps

\begin{tabular}{l|l} 
Score & Criteria Definition \\
\hline 3 & $\begin{array}{l}\text { Data mining methodology or framework is presented in full. All steps described and } \\
\text { explained, tests performed, results compared and evaluated. There is clear proposal on } \\
\text { usage, application, deployment of solution in organization's business process(es) and } \\
\text { IT/IS system, and/or prototype or full solution implementation is discussed. Success } \\
\text { factors described and presented }\end{array}$ \\
$\begin{array}{l}\text { Data mining methodology or framework is presented, some process steps are missing, } \\
\text { but they do not impact the holistic view and understanding of the performed work. Data } \\
\text { mining process is clearly presented and described, tests performed, results compared } \\
\text { and evaluated. There is proposal on usage, application, deployment of solution in } \\
\text { organization's business process(es) and IT/IS system(s) }\end{array}$ \\
$\begin{array}{l}\text { Data mining methodology or framework is not presented in full, some key phases and } \\
\text { process steps are missing. Publication focuses on one or some aspects (eg. method, } \\
\text { technique) }\end{array}$ \\
$\begin{array}{l}\text { Data mining methodology or framework not presented as holistic approach, but on } \\
\text { fragmented basis, study limited to some aspects (eg. method or technique discussion, } \\
\text { etc.) }\end{array}$
\end{tabular}

Table 3. Scoring Metrics

\section{Results and Quantitative Analysis}

In Step 1, 1715 publications were extracted from relevant databases with the following composition Scopus (819), Web of Science (489), Google Scholar (407). In terms of scientific publication domains, Computer Science (42.4\%), Engineering (20.6\%), Mathematics (11.1\%) accounted for app. $74 \%$ of Scopus originated texts. The same applies to Web of Science harvest. Exclusion Criteria application produced the following results. In Step 2, after eliminating duplicates, 1186 texts were passed for minimum length evaluation, and 767 reached assessment by Relevancy Criteria.

As mentioned Relevance Criteria were applied iteratively (Step 4-Step 6) and in conjunction with availability assessment. As a result, only 298 texts were retained for full evaluation with 241 originating from scientific databases while 57 were 'grey'. These studies formed primary texts corpus which was 


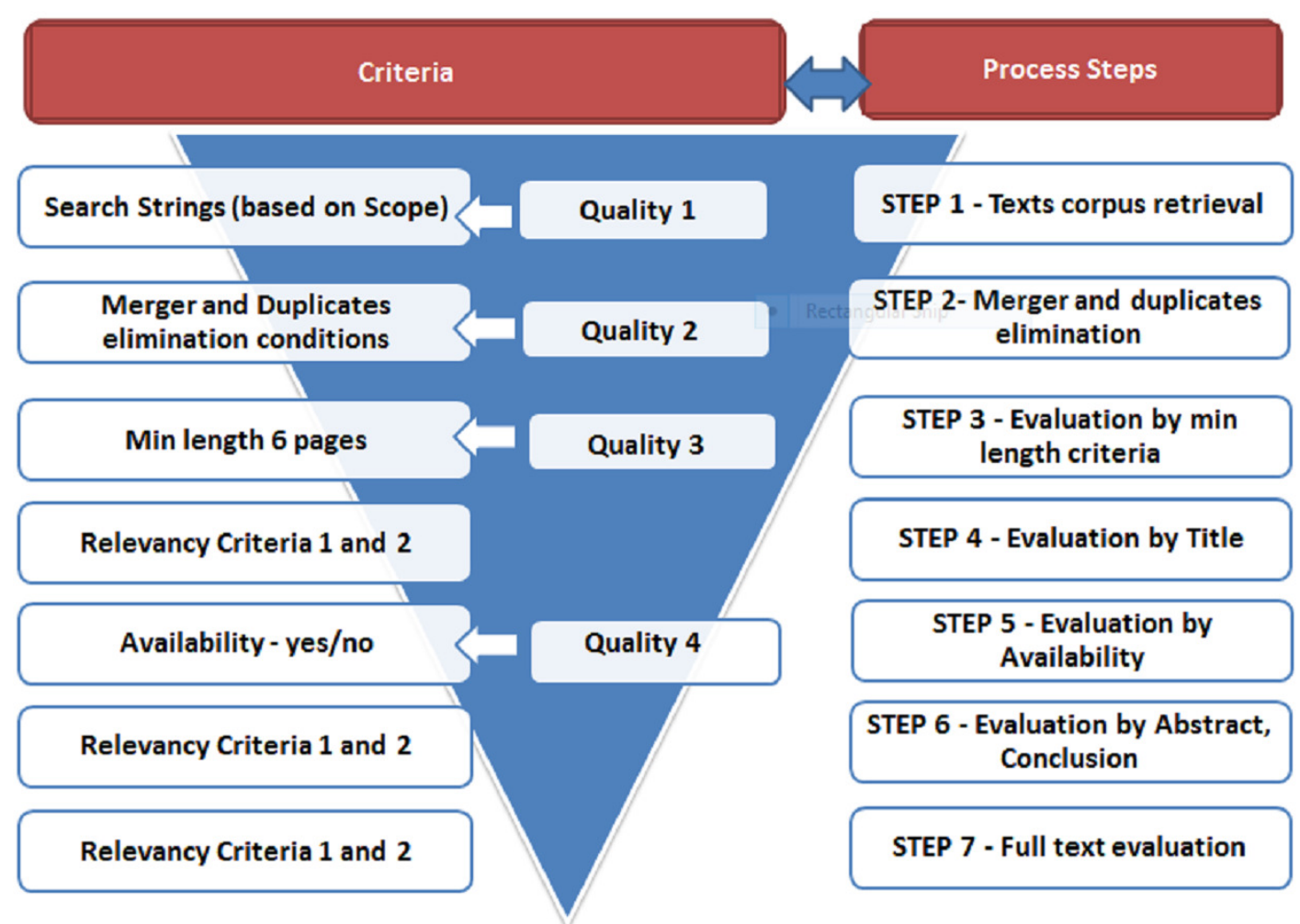

Figure 4. Relevance and Quality Screening Steps with Criteria

extracted, read in full and evaluated by Relevance Criteria combined with Scoring Metrics. The decision rule was set as follows. Studies that scored '1' or '0' were rejected, while texts with '3' and '2' evaluation were admitted as final primary studies corpus. To this end, as an outcome of SLR-based, broad, crossdomain publications collection and screening we identified 207 relevant publications from peer-reviewed (156 texts) and 'grey' literature (51 texts). Figure 5 below exhibits yearly published research numbers with the breakdown by "peer-reviewed" and "grey" literature starting from 1997.

In terms of composition, 'peer-reviewed' studies corpus is well-balanced with 72 journal articles and 82 conference papers while book chapters account for 4 instances only. In contrast, in 'grey' literature subset, articles in moderated and non-peer reviewed journals are dominant $(n=34)$ compared to overall number of conference papers $(n=13)$, followed by small number of technical reports and pre-prints $(n=4)$.

Temporal analysis of texts corpus (as per Figure 5 below) resulted in two observations. Firstly, we note that stable and significant research interest (in terms of numbers) on data mining methodologies application has started around a decade ago - in 2007. Research efforts made prior to 2007 were relatively limited with number of publications below 10. Secondly, we note that research on data mining methodologies has grown substantially since 2007, an observation supported by the 3-year and 10-year constructed mean trendlines. In particular, the number of publications have roughly tripled over past decade hitting all time high with 24 texts released in 2017.

Further, there are also two distinct spike sub-periods in the years 2007-2009 and 2014-2017 followed by stable pattern with overall higher number of released publications on annual basis. This observation is in line with the trend of increased penetration of methodologies, tools, cross-industry applications and academic research of data mining.

\section{FINDINGS AND DISCUSSION}

In this section, we address the research questions of the paper. Initially, as part of RQ1, we present overview of data mining methodologies 'as-is' and adaptation trends. In addressing RQ2, we further classify the adaptations identified. Then, as part of RQ3 subsection, each category identified under RQ2 is analyzed with particular focus on the goals of adaptations. 


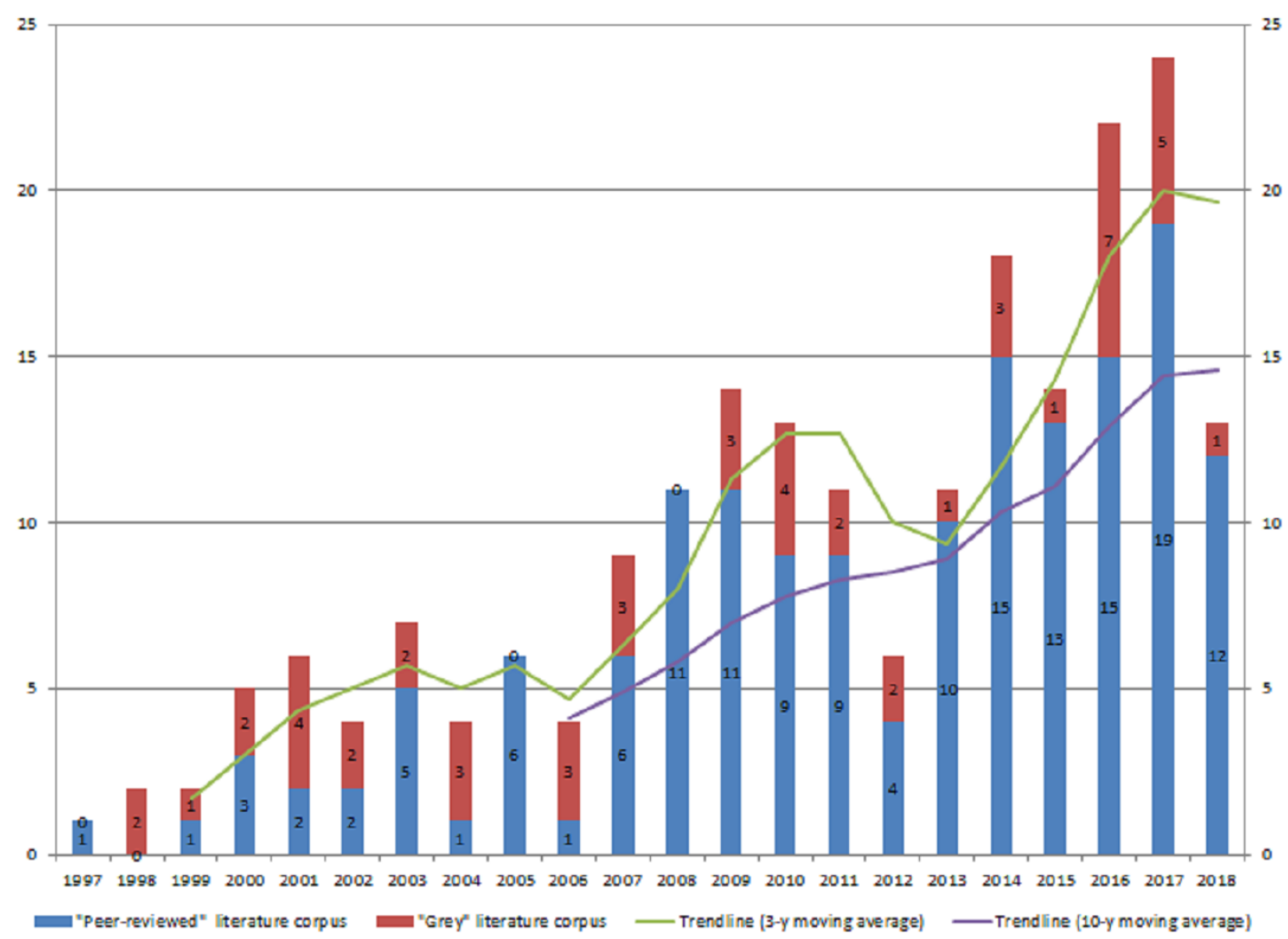

Figure 5. SLR derived relevant texts corpus - data mining methodologies peer-reviewed research and 'grey' for period 1997-2018 (no. of publications).

\section{RQ1: How data mining methodologies are applied ('as-is' vs adapted)?}

The first research question examines the extent to which data mining methodologies are used 'as-is' versus adapted. Our review based on 207 publications identified two distinct paradigms on how data mining methodologies are applied. The first is "as-is" where the data mining methodologies are applied as stipulated. The second is with 'adaptations', i.e., methodologies are modified by introducing various changes to the standard process model when applied.

We have aggregated research by decades to differentiate application pattern between two time periods - 1997-2007 with limited vs 2008-2018 with more intensive data mining application. The given cut has not only been guided by extracted publications corpus but also by earlier surveys. In particular, during the pre-2007 research, there where ten new methodologies proposed, but since then, only two new methodologies have been proposed. Thus, there is a distinct trend observed over the last decade of large number of extensions and adaptations proposed vs entirely new methodologies.

We note that during the first decade of our time scope (1997-2007), the ratio of data mining methodologies applied 'as-is' was 40\% (as presented in Figure 6A). However, the same ratio for the following decade is $32 \%$ (Figure 6B). Thus, in terms of relative shares we note a clear decrease in using data mining methodologies 'as-is' in favour of adapting them to cater to specific needs.The trend is even more pronounced when comparing numbers - adaptations more than tripled (from 30 to 106) while 'as-is' scenario has increased modestly (from 20 to 51). Given this finding, we continue with analyzing how data mining methodologies have been adapted under RQ2.

\section{RQ2: How have existing data mining methodologies been adapted?}

We identified that data mining methodologies have been adapted to cater to specific needs. In order to categorized adaptations scenarios, we applied a two-level dichotomy, specifically, by applying the following decision tree:

1. Level 1 Decision - Has the methodology been combined with another methodology? - If yes, the 

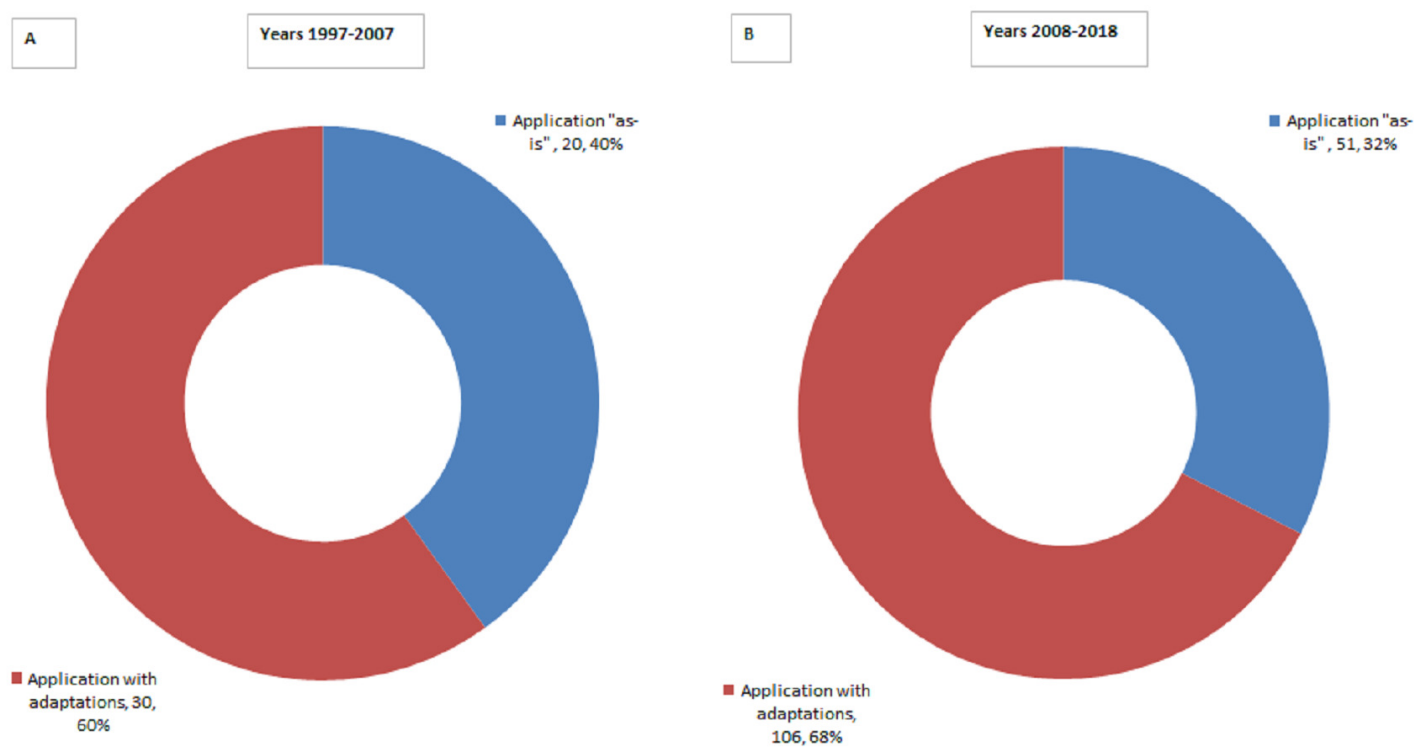

Figure 6. Applications of data mining methodologies: A) breakdown by 'as-is' vs adaptions for 1997-2007 period; B) breakdown by 'as-is' vs adaptions for 2008-2018 period

resulting methodology was classified in the 'integration' category. Otherwise, we posed the next question.

2. Level 2 Decision - Are any new elements (phases, tasks, deliverables) added to the methodology? If yes, we designate the resulting methodology as an "extension" of the original one. Otherwise, we classify the resulting methodology as a modification of the original one.

Thus, when adapted three distinct types of adaptation scenarios can be distinguished:

- Scenario 'Modification' - introduces specialized sub-tasks and deliverables in order to address specific use cases or business problems. Modifications typically concentrate on granular adjustments to the methodology at the level of sub-phases, tasks or deliverables within the existing reference frameworks (eg.CRISP-DM or KDD) stages. For example, Chernov et al. (2014), in the study of mobile network domain, proposed automated decision-making enhancement in the deployment phase. In addition, the evaluation phase was modified by using both conventional and owndeveloped performance metrics. Further, in a study performed within the financial services domain, Yang et al. (2016) presents feature transformation and feature selection as sub-phases, thereby enhancing the data mining modeling stage.

- Scenario 'Extension' - primarily proposes significant extensions to reference data mining methodologies Such extensions result in either integrated data mining solutions, data mining frameworks serving as a component or tool for automated IS systems, or their transformations to fit specialized environments. The main purposes of extensions are to integrate fully-scaled data mining solutions into IS/IT systems and business processes and provide broader context with useful architectures, algorithms, etc. Adaptations, where extensions have been made, elicit and explicitly present various artefacts in the form of system and model architectures, process views, workflows, and implementation aspects. A number of soft goals are also achieved, providing holistic perspective on data mining process, and contextualizing with organizational needs. Also, there are extensions in this scenario where data mining process methodologies are substantially changed and extended in all key phases to enable execution of data mining life-cycle with the new (Big) Data technologies, tools and in new prototyping and deployment environments (eg.Hadoop platforms or real-time customer interfaces). For example, Kisilevich et al. (2013) presented extensions to traditional CRISP-DM data mining outcomes with fully fledged Decision Support System (DSS) for hotel brokerage business. Authors (Kisilevich et al., 2013) have introduced spatial/non-spatial data management 


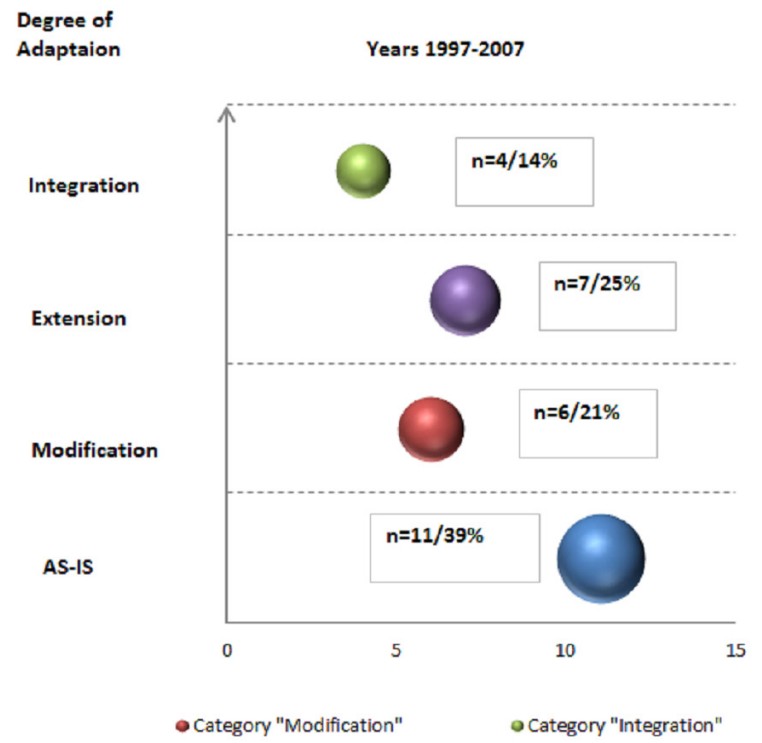
and ease of use were also addressed.

(extending data preparation), analytical and spatial modeling capabilities (extending modeling phase), provided spatial display and reporting capabilities (enhancing deployment phase). In the same work domain knowledge was introduced in all phases of data mining process, and usability

- Scenario 'Integration' - combines reference methodology, eg. CRISP-DM with: (1) data mining methodologies originated from other domains (eg. Software engineering development methodologies), (2) organizational frameworks (Balanced Scorecard, Analytics Canvass, etc.), or (3) adjustments to accommodate Big Data technologies and tools. Also, adaptations in the form of 'Integration' typically introduce various types of ontologies and ontology-based tools, domain knowledge, software engineering, and BI-driven framework elements. Fundamental data mining process adjustments to new types of data, IS architectures (eg. real time data, multi-layer IS) are also presented. Key gaps addressed with such adjustments are prescriptive nature and low degree of formalization in CRISP-DM, obsolete nature of CRISP-DM with respect to tools, and lack of CRISP-DM integration with other organizational frameworks. For example, Brisson and Collard (2008) developed KEOPS data mining methodology (CRIPS-DM based) centered on domain knowledge integration. Ontology-driven information system has been proposed with integration and enhancements to all steps of data mining process. Further, an integrated expert knowledge used in all data mining phases was proved to produce value in data mining process.

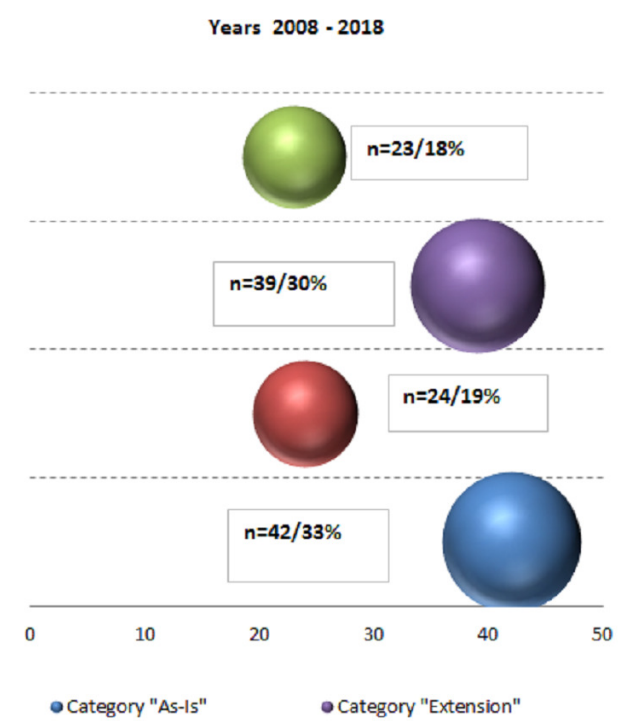

Figure 7. Data Mining methodologies application research - primary 'peer-reviewed' texts classification by types of scenarios aggregated by decades (with numbers and relative proportions)

To examine how the application scenario of each data mining methodology usage has developed over time, we mapped peer-reviewed texts and 'grey' literature to respective adaptation scenarios, aggregated by decades (as presented in the Figure 7 for peer-reviewed and Figure 8 for 'grey').

For peer-reviewed research, such temporal analysis resulted in three observations. Firstly, research efforts in each adaptation scenario has been growing and number of publication more than quadrupled (128 vs 28). Secondly, as noted above relative proportion of 'as-is' studies is diluted (from 39\% to 33\%) and primarily replaced with 'Extension' paradigm (from 25\% to 30\%). In contrast, in relative terms 'Modification' and 'Integration' paradigms gains are modest. Further, this finding is reinforced with other observation - most notable gaps in terms of modest number of publications remain in 'Integration' category where excluding 2008-2009 spike, research efforts are limited and number of texts is just 13. This is in stark contrast with prolific research in 'Extension category' though concentrated in the recent years. We can hypothesize that existing reference methodologies do not accommodate and support increasing complexity of data mining projects and IS/IT infrastructure, as well as certain domains specifics and as 


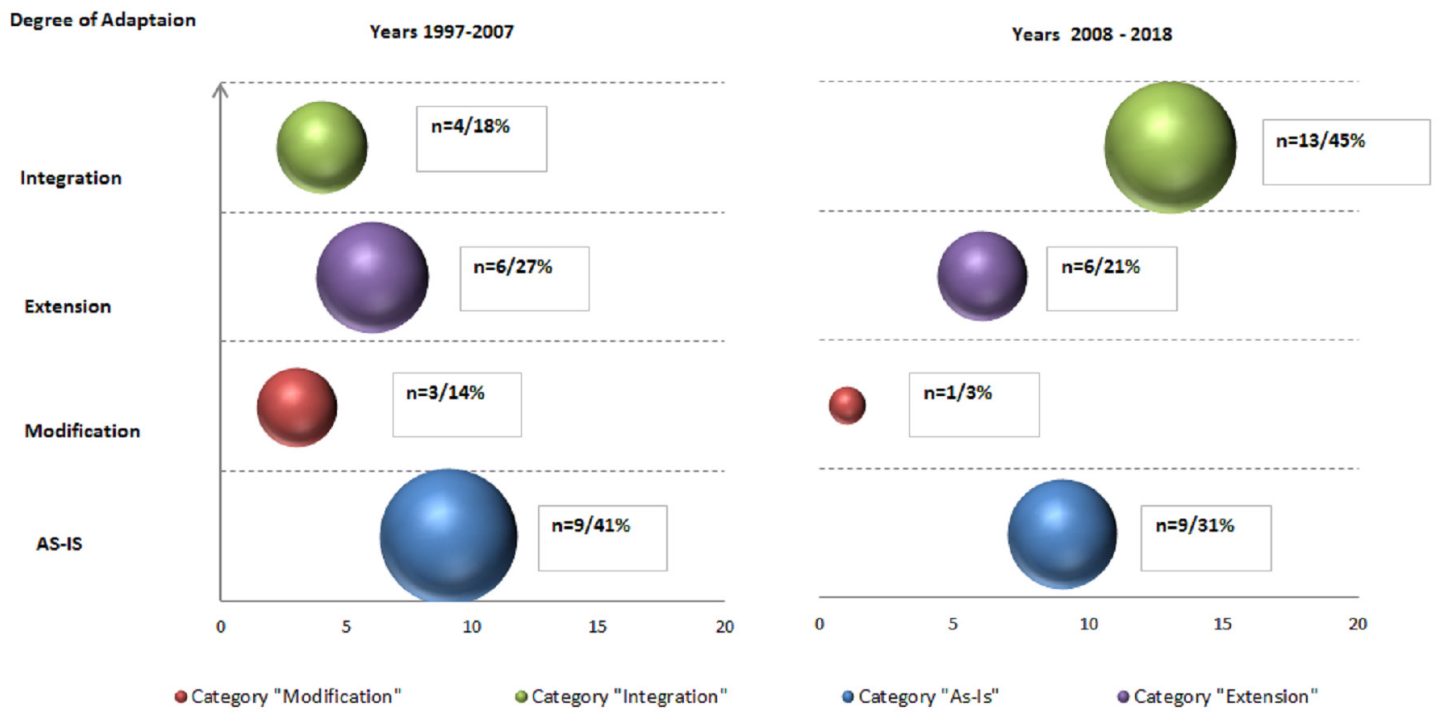

Figure 8. Data Mining methodologies application research - primary 'grey' texts classification by types of scenarios aggregated by decades (with numbers and relative proportions)

such need to be adapted.

In 'grey' literature, in contrast to peer-reviewed research, growth in number of publications is less profound - 29 vs 22 publications or $32 \%$ comparing across two decade (as per Figure 8 ). The growth is solely driven by 'Integration' scenarios application ( 13 vs 4 publications) while both 'as-is' and other adaptations scenarios are stagnating or in decline.

\section{RQ3: For what purposes have existing data mining methodologies been adapted?}

We address the third research question by analyzing what gaps the data mining methodology adaptations seek to fill and the benefits of such adaptations. We identified three adaptation scenarios, namely 'Modification', 'Extension', and 'Integration'. Here, we analyse each of them.

\section{'Modification'}

Modifications of data mining methodologies are present in 30 peer-reviewed and 4 'grey' literature studies. The analysis shows that modifications overwhelmingly consist of specific case studies. However, the major differentiating point compared to 'as-is' case studies is clear presence of specific adjustments towards standard data mining process methodologies. Yet, the proposed modifications and their purposes do not go beyond traditional data mining methodologies phases. They are granular, specialized and executed on tasks, sub-tasks, and at deliverables level. With modifications, authors describe potential business applications and deployment scenarios at a conceptual level, but typically do not report or present real implementations in the IS/IT systems and business processes.

Further, this research subcategory can be best classified based on domains where case studies were performed and data mining methodologies modification scenarios executed. We have identified four distinct domain-driven applications presented in the Figure 9 below.

IT, IS Domain The largest number of publications (14 or app. 40\%), was performed on IT, IS security, software development, specific data mining and processing topics. Authors address intrusion detection problem in Hossain et al. (2003), Fan et al. (2016), Lee et al. (1999), specialized algorithms for variety of data types processing in Yang and Shi (2010), Chen et al. (2001), Yi et al. (2016), Pouyanfar and Chen (2016), effective and efficient computer and mobile networks management in Guan and Fu (2010), Ertek et al. (2017), Zaki and Sobh (2005), Chernov et al. (2015), Chernov et al. (2014).

Manufacturing and Engineering The next most popular research area is manufacturing/engineering with 10 case studies. The central topic here is high-technology manufacturing, eg. semi-conductors associated - study of Chien et al. (2014), and various complex prognostics case studies in rail, aerospace domains (Létourneau et al., 2005), (Zaluski et al., 2011) concentrated on failure predictions. These are 


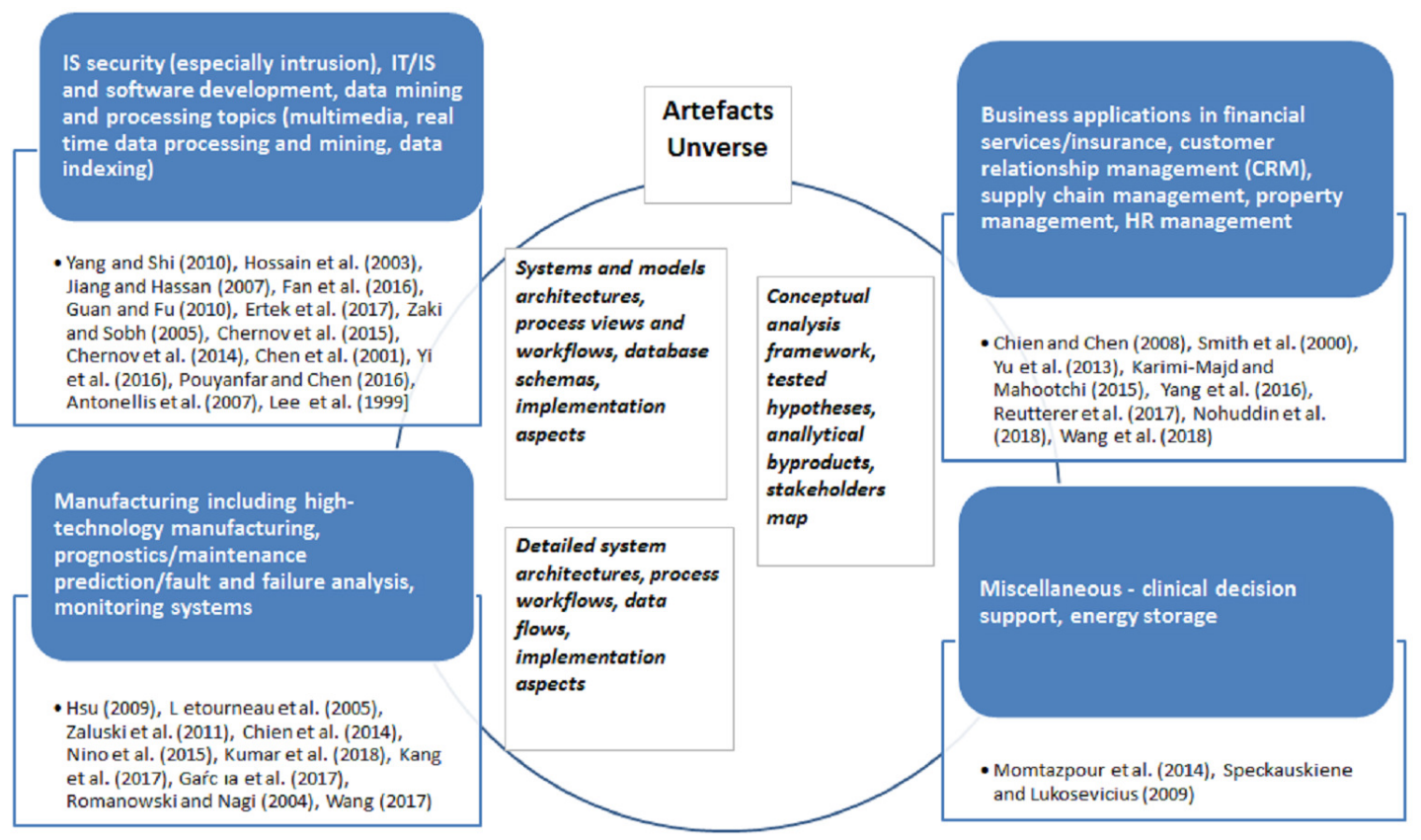

Figure 9. 'Modification' paradigm application studies for period 1997-2018 - mapping to domains

complemented by studies on equipment fault and failure predictions and maintenance (Kumar et al., 2018), (Kang et al., 2017), (Wang, 2017) as well as monitoring system (García et al., 2017).

Sales and Services, incl. Financial Industry The third category is presented by 7 business application papers concerning customer service, targeting and advertising (Karimi-Majd and Mahootchi, 2015), (Reutterer et al., 2017), (Wang, 2017), financial services credit risk assessments (Smith et al., 2000), supply chain management (Nohuddin et al., 2018), and property management (Yu et al., 2013), and similar.

As a consequence of specialization, these studies concentrate on developing 'state-of-the art' solution to the respective domain-specific problem.

\section{'Extension'}

'Extension' scenario was identified in 46 peer-reviewed and 12 'grey' publications. We noted that 'Extension' to existing data mining methodologies were executed with four major purposes:

1. Purpose 1 - To implement fully scaled, integrated data mining solution and regular, repeatable knowledge discovery process - address model, algorithm deployment, implementation design (including architecture, workflows and corresponding IS integration). Also, complementary goal is to tackle changes to business process to incorporate data mining into organization activities.

2. Purpose 2 - To implement complex, specifically designed systems and integrated business applications with data mining model/solution as component or tool. Typically, this adaptation is also oriented towards Big Data specifics, and is complemented by proposed artefacts such as Big Data architectures, system models, workflows, and data flows.

3. Purpose 3 - To implement data mining as part of integrated/combined specialized infrastructure, data environments and types (eg. IoT, cloud, mobile networks).

\section{Purpose 4 - To incorporate context-awareness aspects.}

The specific list of studies mapped to each of the given purposes presented in the Appendix, Table A1. Main purposes of adaptations, associated gaps and/or benefits along with observations and artefacts are documented in the Figure 10 below.

In 'Extension' category, studies executed with the Purpose 1 propose fully scaled, integrated data mining solutions of specific data mining models, associated frameworks and processes. The distinctive 


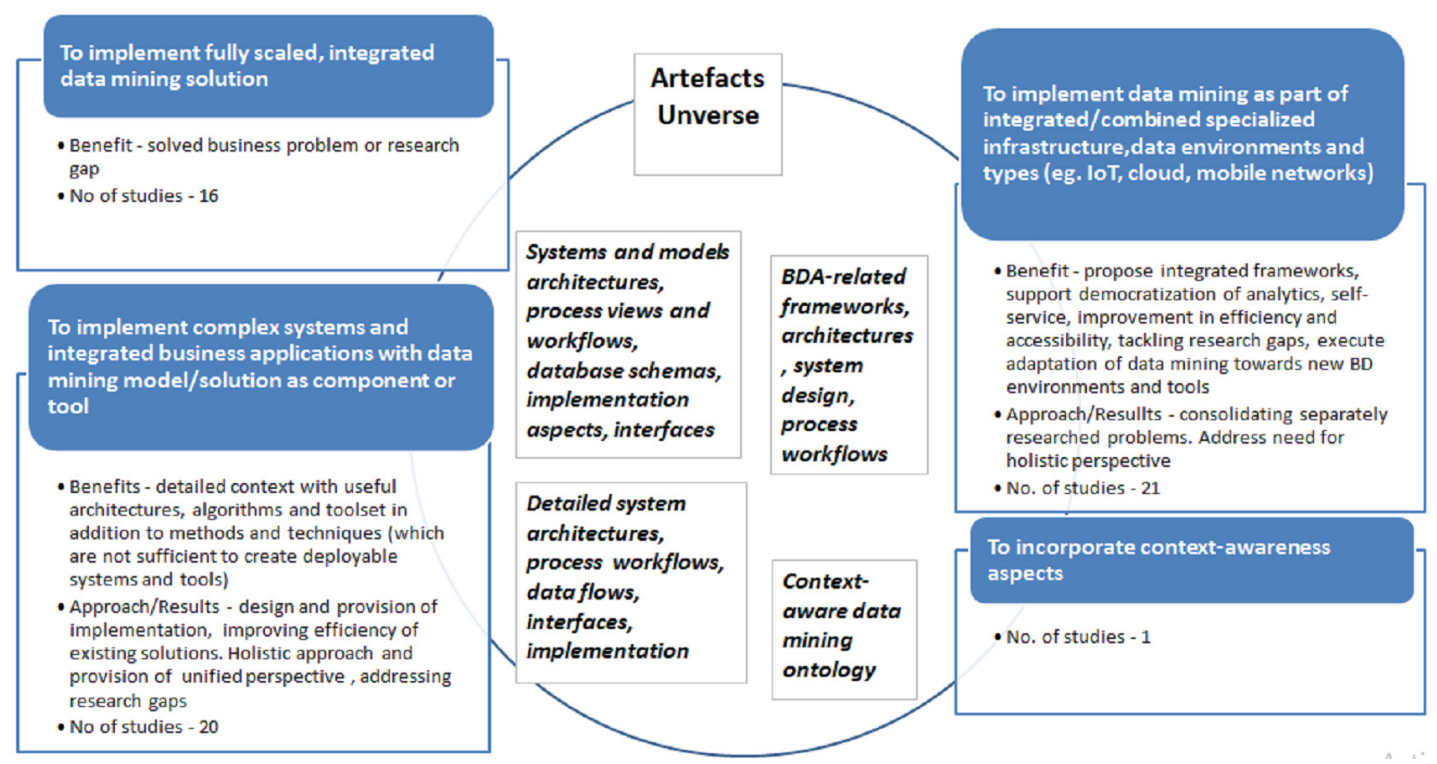

Figure 10. 'Extension' scenario adaptations goals, benefits, artefacts and number of publications for period 1997-2018

trait of this research subclass is that it ensures repeatability and reproducibility of delivered data mining solution in different organizational and industry settings. Both the results of data mining use case as well as deployment and integration into IS/IT systems and associated business process(es) are presented explicitly. Thus, 'Extension' subclass is geared towards specific solution design, tackling concrete business or industrial setting problem or addressing specific research gaps thus resembling comprehensive case study.

This direction can be well exemplified by expert finder system in research social network services proposed by Sun et al. (2015), data mining solution for functional test content optimization by Wang (2015) and time-series mining framework to conduct estimation of unobservable time-series by $\mathrm{Hu}$ et al. (2010). Similarly, Du et al. (2017) tackle online log anomalies detection, automated association rule mining is addressed by Çinicioğlu et al. (2011), software effort estimation by Deng et al. (2011), network patterns visual discovery by Simoff and Galloway (2008). Number of studies address solutions in IS security (Shin and Jeong, 2005), manufacturing (Güder et al., 2014), (Chee et al., 2016), materials engineering domains (Doreswamy, 2008), and business domains (Xu and Qiu, 2008), (Ding and Daniel, 2007).

In contrast, 'Extension' studies executed for the Purpose 2 concentrate on design of complex, multicomponent information systems and architectures. These are holistic, complex systems and integrated business applications with data mining framework serving as component or tool. Moreover, data mining methodology in these studies is extended with systems integration phases.

For example, Mobasher (2007) presents data mining application in Web personalization system and associated process; here, data mining cycle is extended in all phases with utmost goal of leveraging multiple data sources and using discovered models and corresponding algorithms in an automatic personalization system. Authors comprehensively address data processing, algorithm, design adjustments and respective integration into automated system. Similarly, Haruechaiyasak et al. (2004) tackle improvement of Webpage recommender system by presenting extended data mining methodology including design and implementation of data mining model. Holistic view on web-mining with support of all data sources, data warehousing and data mining techniques integration, as well as multiple problem-oriented analytical outcomes with rich business application scenarios (personalization, adaptation, profiling, and recommendations) in e-commerce domain was proposed and discussed by Büchner and Mulvenna (1998). Further, Singh et al. (2014) tackled scalable implementation of Network Threat Intrusion Detection System. In this study, data mining methodology and resulting model are extended, scaled and deployed as module of quasi-real-time system for capturing Peer-to-Peer Botnet attacks. Similar complex solution was presented in a series of publications by Lee et al. (2000) and Lee et al. (2001) who designed real-time 
data mining-based Intrusion Detection System (IDS). These works are complemented by comprehensive study of Barbará et al. (2001) who constructed experimental testbed for intrusion detection with data mining methods. Detection model combining data fusion and mining and respective components for Botnets identification was developed by Kiayias et al. (2009) too. Similar approach is presented in Alazab et al. (2011) who proposed and implemented zero-day malware detection system with associated machine-learning based framework. Finally, Ahmed et al. (2011) presented multi-layer framework for fuzzy atack in $3 \mathrm{G}$ cellular IP networks.

A number of authors have considered data mining methodologies in the context of Decision Support Systems and other systems that generate information for decision-making, across a variety of domains. For example, Kisilevich et al. (2013) executed significant extension of data mining methodology by designing and presenting integrated Decision Support System (DSS) with six components acting as supporting tool for hotel brokerage business to increase deal profitability. Similar approach is undertaken by Capozzoli et al. (2017) focusing on improving energy management of properties by provision of occupancy pattern information and reconfiguration framework. Kabir (2016) presented data mining information service providing improved sales forecasting that supported solution of under/over-stocking problem while Lau et al. (2018) addressed sales forecasting with sentiment analysis on Big Data. Kamrani et al. (2001) proposed GA-based Intelligent Diagnosis system for fault diagnostics in manufacturing domain. The latter was tackled further in Shahbaz et al. (2010) with complex, integrated data mining system for diagnosing and solving manufacturing problems in real time.

Lenz et al. Lenz et al. (2018) propose a framework for capturing data analytics objectives and creating holistic, cross-departmental data mining systems in the manufacturing domain. This work is representative of a cohort of studies that aim at extending data mining methodologies in order to support the design and implementation of enterprise-wide data mining systems. In this same research cohort, we classify Luna et al. (2017), which presents a data mining toolset integrated into the Moodle learning management system, with the aim of supporting university-wide learning analytics.

One study addresses Multi-Agent based data mining concept. Khan et al. (2013) have developed unified theoretical framework for data mining by formulating a unified data mining theory. The framework is tested by means of agent programming proposing integration into Multi-Agent System which is useful due to scalability, robustness and simplicity.

The subcategory of 'Extension' research executed with Purpose 3 is devoted to data mining methodologies and solutions in specialized IT/IS, data and process environments which emerged recently as consequence of Big Data associated technologies and tools development. Exemplary studies include IoT associated environment research, for example, Smart City application in IoT presented by Strohbach et al. (2015). In the same domain, Bashir and Gill (2016) addressed IoT-enabled smart buildings with the additional challenge of large amount of high-speed real time data and requirements of real-time analytics. Authors proposed integrated IoT Big Data Analytics framework. This research is complemented by interdisciplinary study of Zhong et al. (2017) where IoT and wireless technologies are used to create RFID-enabled environment producing analysis of KPIs to improve logistics.

Significant number of studies addresses various mobile environments sometimes complemented by cloud-based environments or cloud-based environments as stand-alone. Gomes et al. (2013) addressed mobile data mining with execution on mobile device itself; the framework proposes innovative approach addressing extensions of all aspects of data mining including contextual data, end-user privacy preservation, data management and scalability. Yuan et al. (2014) and Yuan and Herbert (2014) introduced cloudbased mobile data analytics framework with application case study for smart home based monitoring system. Cuzzocrea et al. (2016) have presented innovative FollowMe suite which implements data mining framework for mobile social media analytics with several tools with respective architecture and functionalities. Interesting paper was presented by Torres et al. (2017) who addressed data mining methodology and its implementation for congestion prediction in mobile LTE networks tackling also feedback reaction with network reconfigurations trigger.

Further, Biliri et al. (2014) presented cloud-based Future Internet Enabler - automated social data analytics solution which also addresses Social Network Interoperability aspect supporting enterprises to interconnect and utilize social networks for collaboration. Real-time social media streamed data and resulting data mining methodology and application was extensively discussed by Zhang et al. (2014). Authors proposed design of comprehensive ABIGDAD framework with seven main components implementing data mining based deceptive review identification. Interdisciplinary study tackling both these 
topics was developed by Puthal et al. (2016) who proposed integrated framework and architecture of disaster management system based on streamed data in cloud environment ensuring end-to-end security. Additionally, key extensions to data mining framework have been proposed merging variety of data sources and types, security verification and data flow access controls. Finally, cloud-based manufacturing was addressed in the context of fault diagnostics by Kumar et al. (2016).

Also, Mahmood et al. (2013) tackled Wireless Sensor Networks and associated data mining framework required extensions. Interesting work is executed by Nestorov and Jukic (2003) addressing rare topic of data mining solutions integration within traditional data warehouses and active mining of data repositories themselves.

Supported by new generation of visualization technologies (including Virtual Reality environments), Wijayasekara et al. (2011) proposed and implemented CAVE-SOM (3D visual data mining framework) which offers interactive, immersive visual data mining with multiple visualization modes supported by plethora of methods. Earlier version of visual data mining framework was successfully developed and presented by Ganesh et al. (1996) as early as in 1996.

Large-scale social media data is successfully tackled by Lemieux (2016) with comprehensive framework accompanied by set of data mining tools and interface. Real time data analytics was addressed by Shrivastava and Pal (2017) in the domain of enterprise service ecosystem. Images data was addressed in Huang et al. (2002) by proposing multimedia data mining framework and its implementation with user relevance feedback integration and instance learning. Further, exploded data diversity and associated need to extend standard data mining is addressed by Singh et al. (2016) in the study devoted to object detection in video surveillance systems supporting real time video analysis.

Finally, there is also limited number of studies which addresses context awareness (Purpose 4) and extends data mining methodology with context elements and adjustments. In comparison with 'Integration' category research, here, the studies are at lower abstraction level, capturing and presenting list of adjustments. Singh et al. (2003) generate taxonomy of context factors, develop extended data mining framework and propose deployment including detailed IS architecture. Context-awareness aspect is also addressed in the papers reviewed above, eg. Lenz et al. (2018), Kisilevich et al. (2013), Sun et al. (2015), and other studies.

\section{'Integration'}

'Integration' of data mining methodologies scenario was identified in 27 'peer-reviewed' and 17 'grey' studies. Our analysis revealed that this adaptation scenario at a higher abstraction level is typically executed with the 5 key purposes:

\section{Purpose 1 - to integrate/combine with various ontologies existing in organization.}

2. Purpose 2 - to introduce context-awareness and incorporate domain knowledge.

3. Purpose 3 - to integrate/combine with other research or industry domains framework, process methodologies and concepts.

4. Purpose 4 - to integrate/combine with other well-known organizational governance frameworks, process methodologies and concepts.

5. Purpose 5 - to accommodate and/or leverage upon newly available Big Data technologies, tools and methods.

The specific list of studies mapped to each of the given purposes presented in Appendix, Table A2. Main purposes of adaptations, associated gaps and/or benefits along with observations and artefacts are documented in Figures 11 below.

As mentioned, number of studies concentrates on proposing ontology-based Integrated data mining frameworks accompanies by various types of ontologies (Purpose 1). For example, Sharma and OseiBryson (2008) focus on ontology-based organizational view with Actors, Goals and Objectives which supports execution of Business Understanding Phase. Brisson and Collard (2008) propose KEOPS framework which is CRISP-DM compliant and integrates a knowledge base and ontology with the purpose to build OIS (ontology-driven information system) for business and data understanding phases while knowledge base is used for post-processing step of model interpretation. Park et al. (2017) propose and design comprehensive ontology-based data analytics tool IRIS with the purpose to align analytics 


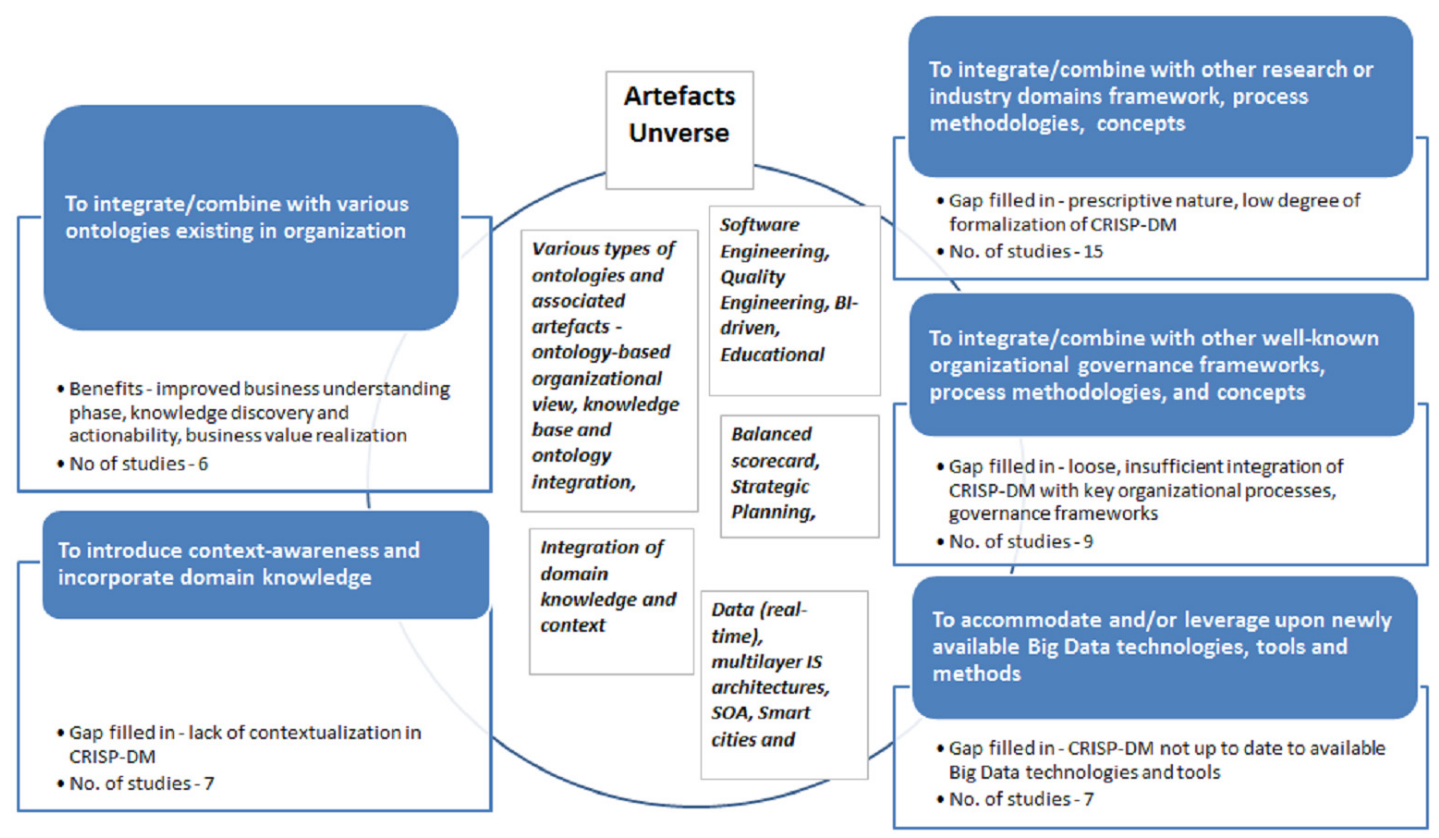

Figure 11. 'Integration' scenario adaptations goals, benefits, artefacts and number of publications for period 1997-2018

and business. IRIS is based on concept to connect dots, analytics methods or transforming insights into business value, and supports standardized process for applying ontology to match business problems and solutions.

Further, Ying et al. (2014) propose domain-specific data mining framework oriented to business problem of customer demand discovery. They construct ontology for customer demand and customer demand discovery task which allows to execute structured knowledge extraction in the form of knowledge patterns and rules. Here, the purpose is to facilitate business value realization and support actionability of extracted knowledge via marketing strategies and tactics. In the same vein, Cannataro and Comito (2003) presented ontology for the Data Mining domain which main goal is to simplify the development of distributed knowledge discovery applications. Authors offered to a domain expert a reference model for different kind of data mining tasks, methodologies, and software capable to solve the given business problem and find the most appropriate solution.

Apart from ontologies, Sharma and Osei-Bryson (2009) in another study propose IS inspired, driven by Input-Output model data mining methodology which supports formal implementation of Business Understanding Phase. This research exemplifies studies executed with Purpose 2. The goal of the paper is to tackle prescriptive nature of CRISP-DM and address how the entire process can be implemented. Cao et al. (2005) study is also exemplary in terms of aggregating and introducing several fundamental concepts into traditional CRISP-DM data mining cycle - context awareness, in-depth pattern mining, human-machine cooperative knowledge discovery (in essence, following human-centricity paradigm in data mining), loop-closed iterative refinement process (similar to Agile-based methodologies in Software Development). There are also several concepts, like data, domain, interestingness, rules which are proposed to tackle number of fundamental constrains identified in CRISP-DM. They have been discussed and further extended by Cao and Zhang (2007), Cao and Zhang (2008), Cao (2010) into integrated domain driven data mining concept resulting in fully fledged D3M (domain-driven) data mining framework. Interestingly, the same concepts, but on individual basis are investigated and presented by other authors, eg. context-aware data mining methodology is tackled by Xiang (2009a), Xiang (2009b) in the context of financial sector. Pournaras et al. (2016) attempted very crucial privacy-preservation topic in the context of achieving effective data analytics methodology. Authours introduced metrics and self-regulatory (reconfigurable) information sharing mechanism providing customers with controls for information disclosure.

A number of studies have proposed CRISP-DM adjustments based on existing frameworks, process 
models or concepts originating in other domains (Purpose 3), for example, software engineering Marbán et al. (2007); Marbán et al. (2009); Marban et al. (2009) and industrial engineering Solarte (2002); Zhao et al. (2005).

Meanwhile, Mariscal et al. (2010) proposed a new refined data mining process based on a global comparative analysis of existing frameworks while Angelov (2014) outlined a data analytics framework based on statistical concepts. Following a similar approach, some researchers suggest explicit integration with other areas and organizational functions, for example, BI-driven Data Mining by Hang and Fong (2009). Similarly, Chen et al. (2016) developed an architecture-centric agile Big Data analytics methodology, and an architecture-centric agile analytics and DevOps model. Alternatively, several authors tackled data mining methodology adaptations in other domains, e.g. educational data mining by Tavares et al. (2017), decision support in learning management systems (Murnion and Helfert, 2011), and in accounting systems (Amani and Fadlalla, 2017).

Other studies are concerned with actionability of data mining and closer integration with business processes and organizational management frameworks (Purpose 4). In particular, there is a recurrent focus on embedding data mining solutions into knowledge-based decision making processes in organizations, and supporting fast and effective knowledge discovery (Bohanec et al., 2017).

Examples of adaptations made for this purpose include: (1) integration of CRISP-DM with the Balanced Scorecard framework used for strategic performance management in organizations (Yun et al., 2014); (2) integration with a strategic decision-making framework for revenue management Segarra et al (2016); (3) integration with a strategic analytics methodology van Rooyen and Simoff (2008), and (4) integration with a so-called 'Analytics Canvas' for management of portfolios of data analytics projects Kühn et al. (2018). Finally, Ahangama and Poo (2015) explored methodological attributes important for adoption of data mining methodology by novice users. This latter study uncovered factors that could support the reduction of resistance to the use of data mining methodologies. Conversely, Lawler and Joseph (2017) comprehensively evaluated factors that may increase the benefits of Big Data Analytics projects in an organization.

Lastly, a number of studies have proposed data mining frameworks, (eg. CRISP-DM) adaptations to cater for new technological architectures, new types of datasets and applications (Purpose 5). For example, Lu et al. (2017) proposed a data mining system based on a Service-Oriented Architecture (SOA), Zaghloul et al. (2013) developed a concept of self-service data analytics, Osman et al. (2017) blended CRISP-DM into a Big Data Analytics framework for Smart Cities, and Niesen et al. (2016) proposed a data-driven risk management framework for Industry 4.0 applications.

Our analysis of RQ3, regarding the purposes of existing data mining methodologies adaptations, revealed the following key findings. Firstly, adaptations of type 'Modification' are predominantly targeted at addressing problems that are specific to a given case study. The majority of modifications were made within the domain of IS security, followed by case studies in the domains of manufacturing and financial services. This is in clear contrast with adaptations of type 'Extension', which are primarily aimed at customizing the methodology to take into account specialized development environments and deployment infrastructures, and to incorporate context-awareness aspects. Thirdly, a recurrent purpose of adaptations of type 'Integration' is to combine a data mining methodology with either existing ontologies in an organization or with other domain frameworks, methodologies, and concepts. 'Integration' is also used to instill context-awareness and domain knowledge into a data mining methodology, or to adapt it to specialized methods and tools, such as Big Data. The distinctive outcome and value (gaps filled in) of 'Integrations' stems from improved knowledge discovery, better actionability of results, improved combination with key organizational processes and domain-specific methodologies, and improved usage of Big Data technologies.

\section{Summary}

We discovered that the adaptations of existing data mining methodologies found in the literature can be classified into three categories: modification, extension, or integration.

We also noted that adaptations are executed either to address deficiencies and lack of important elements or aspects in the reference methodology (chiefly CRISP-DM). Furthermore, adaptations are also made to improve certain phases, deliverables or process outcomes.

In short, adaptations are made to:

- improve key reference data mining methodologies phases - for example, in case of CRISP-DM 
these are primarily business understanding and deployment phases.

- support knowledge discovery and actionability.

- introduce context-awareness and higher degree of formalization.

- integrate closer data mining solution with key organizational processes and frameworks.

- significantly update CRISP-DM with respect to Big Data technologies, tools, environments and .

- incorporate broader, explicit context of architectures, algorithms and toolsets as integral deliverables or supporting tools to execute data mining process.

- expand and accommodate broader unified perspective for incorporating and implementing data mining solutions in organization, IT infrastructure and business processes.

\section{THREATS TO VALIDITY}

Systematic literature reviews have inherent limitations that must be acknowledged. These threats to validity include subjective bias (internal validity) and incompleteness of search results (external validity).

The internal validity threat stems from the subjective screening and rating of studies, particularly when assessing the studies with respect to relevance and quality criteria. We have mitigated these effects by documenting the survey protocol (SLR Protocol), strictly adhering to the inclusion criteria, and performing significant validation procedures, as documented in the Protocol.

The external validity threat relates to the extent to which the findings of the SLR reflect the actual state of the art in the field of data mining methodologies, given that the SLR only considers published studies that can be retrieved using specific search strings and databases. We have addressed this threat to validity by conducting trial searches to validate our search strings in terms of their ability to identify relevant papers that we knew about beforehand. Also, the fact that the searches led to 1700 hits overall suggests that a significant portion of the relevant literature has been covered.

\section{CONCLUSION}

In this study, we have examined the use of data mining methodologies by means of a systematic literature review covering both peer-reviewed and 'grey' literature. We have found that the use of data mining methodologies, as reported in the literature, has grown substantially since 2007 (four-fold increase relative to the previous decade). Also, we have observed that data mining methodologies were predominantly applied 'as-is' from 1997 to 2007. This trend was reversed from 2008 onward, when the use of adapted data mining methodologies gradually started to replace 'as-is' usage.

The most frequent adaptations have been in the 'Extension' category. This category refers to adaptations that imply significant changes to key phases of the reference methodology (chiefly CRISP-DM). These adaptations particularly target the business understanding, deployment and implementation phases of CRISP-DM (or other methodologies). Moreover, we have found that the most frequent purposes of adaptions are: (1) adaptations to handle Big Data technologies, tools and environments (technological adaptations); and (2) adaptations for context-awareness and for integrating data mining solutions into business processes and IT systems (organizational adaptations).

A key finding is that standard data mining methodologies do not pay sufficient attention to deployment aspects required to scale and transform data mining models into software products integrated into large IT/IS systems and business processes. Apart from the adaptations in the 'Extension' category, we have also identified an increasing number of studies focusing on the 'Integration' of data mining methodologies with other domain-specific and organizational methodologies, frameworks, and concepts. These adaptions are aimed at embedding the data mining methodology into broader organizational aspects.

Overall, the findings of the study highlight the need to develop refinements of existing data mining methodologies that would allow them to seamlessly interact with IT development platforms and processes (technological adaptation) and with organizational management frameworks (organizational adaptation). In other words, there is a need to frame existing data mining methodologies as being part of a broader ecosystem of methodologies, as opposed to the traditional view where data mining methodologies are defined in isolation from broader IT systems engineering and organizational management methodologies. 
Main Adaptation Purpose 1) To implement fully scaled, integrated data mining solution

2) To implement complex systems and integrated business applications with data mining model/solution as component or tool

3) To implement data mining as part of integrated/combined specialized infrastructure, data environments and types (eg. IoT, cloud, mobile networks)

4) To incorporate contextawareness aspects
Publications

Sun et al. (2015), Hu et al. (2010), Wang (2015), Du

et al. (2017), Çinicioğlu et al. (2011), Doreswamy (2008), Güder et al. (2014), Simoff and Galloway (2008), Deng et al. (2011), Xu and Qiu (2008), Shin and Jeong (2005), Chee et al. (2016), Yu et al. (2009), Ding and Daniel (2007), Liu et al. (2018), Shao et al. (2008)

Mobasher (2007), Singh et al. (2014), Alazab et al. (2011), Kisilevich et al. (2013), Haruechaiyasak et al. (2004), Luna et al. (2017), Khan et al. (2013), Ortega et al. (2015), Lau et al. (2018), Ahmed et al. (2011), Capozzoli et al. (2017), Kabir (2016), Kiayias et al. (2009), Kamrani et al. (2001), Büchner and Mulvenna (1998), Shahbaz et al. (2010), Lee et al. (2001), Lee et al. (2000), Barbará et al. (2001), Lenz et al. (2018)

Strohbach et al. (2015), Mahmood et al. (2013), Nestorov and Jukic (2003), Gomes et al. (2013), Wijayasekara et al. (2011), Yuan and Herbert (2014), Bashir and Gill (2016), Cuzzocrea et al. (2016), Biliri et al. (2014), Rendall et al. (2017), Zhang et al. (2014), Yuan et al. (2014), Huang et al. (2002), Singh et al. (2016), Shrivastava and Pal (2017), Lemieux (2016), Ganesh et al. (1996), Torres et al. (2017), Zhong et al. (2017), Puthal et al. (2016), Kumar et al. (2016)

Singh et al. (2003)

Table A1. 'Extension' paradigm data mining methodologies application studies for period 1997-2018 


\begin{tabular}{|c|c|}
\hline Main Adaptation Purpose & Publications \\
\hline $\begin{array}{l}\text { 1) To integrate/combined with } \\
\text { various ontologies existing in or- } \\
\text { ganization }\end{array}$ & $\begin{array}{l}\text { Sharma and Osei-Bryson (2008), Sharma and Osei-Bryson } \\
\text { (2009), Brisson and Collard (2008), Park et al. (2017), } \\
\text { Ying et al. (2014) Cannataro and Comito (2003) }\end{array}$ \\
\hline $\begin{array}{l}\text { 2) To introduce context- } \\
\text { awareness and incorporate } \\
\text { domain knowledge }\end{array}$ & $\begin{array}{l}\text { Cao et al. (2005), Cao and Zhang (2008), Xiang (2009a), } \\
\text { Xiang (2009b), Pournaras et al. (2016), Cao and Zhang } \\
(2007) \text {, Cao (2010) }\end{array}$ \\
\hline $\begin{array}{l}\text { 3) To integrate/combine with } \\
\text { other research/industry domains } \\
\text { frameworks, process methodolo- } \\
\text { gies, and concepts }\end{array}$ & $\begin{array}{l}\text { Marbán et al. (2007), Zhao et al. (2005), François (2008), } \\
\text { Hang and Fong (2009), Tavares et al. (2017), Murnion and } \\
\text { Helfert (2011), Amani and Fadlalla (2017), Marban et al. } \\
\text { (2009), Mariscal et al. (2010), Solarte (2002), Marbán et al. } \\
\text { (2009), Chen et al. (2016), Ahangama and Poo (2015), } \\
\text { Angelov (2014) }\end{array}$ \\
\hline $\begin{array}{l}\text { 4) To integrate/combine with } \\
\text { other organizational governance } \\
\text { frameworks, process methodolo- } \\
\text { gies, concepts }\end{array}$ & $\begin{array}{l}\text { Bohanec et al. (2017), Debuse (2007), Chatzikonstantinou } \\
\text { et al. (2013), Rahman et al. (2011), Yun et al. (2014), van } \\
\text { Rooyen and Simoff (2008), Kühn et al. (2018), Segarra } \\
\text { et al. (2016), Lawler and Joseph (2017) }\end{array}$ \\
\hline $\begin{array}{l}\text { 5) To accomodate or leverage } \\
\text { upon newly available Big Data } \\
\text { technologies, tools and methods }\end{array}$ & $\begin{array}{l}\text { Lu et al. (2017), Osman et al. (2017), Behbahani et al. } \\
\text { (2011), Deng et al. (2009), Kurgan and Musílek (2006),Za- } \\
\text { ghloul et al. (2013), Niesen et al. (2016) }\end{array}$ \\
\hline
\end{tabular}

Table A2. 'Integration' paradigm data mining methodologies application studies for period 1997-2018 


\section{REFERENCES}

Adrian, C., Sidi, F., Abdullahm, R., Ishak, I., Suriani, A., L., and Jabar, A., A. (2004). Big data analytics implementation for value discovery: A systematic literature review. Journal of Theoretical and Applied Information Technology, 93(2):385-393.

Anand, S. S. and Büchner, A. G. (1998). Decision support using data mining. Financial Times Management.

Anand, S. S., Patrick, A., Hughes, J. G., and Bell, D. A. (1998). A data mining methodology for cross-sales. Knowledge-based systems, 10(7):449-461.

Bi, Z. and Cochran, D. (2014). Big data analytics with applications. Journal of Management Analytics, 1(4):249-265.

Blockeel, H. and Moyle, S. (2002). Collaborative data mining needs centralised model evaluation. In Proceedings of the ICML-2002 Workshop on Data Mining Lessons Learned, pages 21-28.

Bose, I. and Mahapatra, R. K. (2001). Business data mining - a machine learning perspective. Information \& Management, 39(3):211-225.

Brachman, R. J. and Anand, T. (1996). The process of knowledge discovery in databases. In Advances in Knowledge Discovery and Data Mining, pages 37-57. American Association for Artificial Intelligence.

Brereton, P., Kitchenham, B. A., Budgen, D., Turner, M., and Khalil, M. (2007). Lessons from applying the systematic literature review process within the software engineering domain. Journal of Systems and Software, 80(4):571-583.

Buchner, A. G., Mulvenna, M. D., Anand, S. S., and Hughes, J. G. (1999). An internet-enabled knowledge discovery process. In Proceedings of the 9th international database conference, Hong Kong, volume 1999, pages 13-27.

Budgen, D., Charters, S. M., Turner, M., Brereton, P., Kitchenham, B. A., and Linkman, S. G. (2006). Investigating the applicability of the evidence-based paradigm to software engineering. In Proceedings of the 2006 international workshop on Workshop on interdisciplinary software engineering research, WISER 2006, Shanghai, China, May 20, 2006, pages 7-14.

Cabena, P., Hadjinian, P., Stadler, R., Verhees, J., Zanasi, A., International Business Machines Corporation (San Jose, C., and International Technical Support Organization (San Jose, C. (1997). Discovering data mining: from concept to implementation. Prentice Hall PTR New Jersey.

Chapman, P., Clinton, J., Kerber, R., Khabaza, T., Reinartz, T., Shearer, C., and Wirth, R. (2000). Crisp-dm 1.0 step-by-step data mining guide. SPSSInc.

Chen, H., Chiang, R. H. L., and Storey, V. C. (2012). Business intelligence and analytics: From big data to big impact. MIS Quarterly, 36(4):1165-1188.

Cios, K. J. and Kurgan, L. A. (2005). Trends in data mining and knowledge discovery. In Advanced techniques in knowledge discovery and data mining, pages 1-26. Springer.

Columbus, L. (Dec 24, 2017). Forbes homepage. 53\% Of Companies Are Adopting Big Data Analytics, https://www.forbes.com/sites/louiscolumbus/2017/12/24/53-of-companies-are-adopting-big-dataanalytics/4cf12a2139a1, last accessed 2019/10/26.

de Pisón Ascacíbar, F. J. M. (2003). Optimización mediante técnicas de minería de datos del ciclo de recocido de una línea de galvanizado. Universidad de La Rioja.

Debuse, J., de la Iglesia, B., Howard, C., and Rayward-Smith, V. (2001). Building the kdd roadmap. In Industrial Knowledge Management, pages 179-196. Springer.

Fayyad, U. M., Piatetsky-Shapiro, G., and Smyth, P. (1996a). From data mining to knowledge discovery in databases. AI Magazine, 17(3):37-54. 
Fayyad, U. M., Piatetsky-Shapiro, G., and Smyth, P. (1996b). The KDD process for extracting useful knowledge from volumes of data. Commun. ACM, 39(11):27-34.

Fayyad, U. M., Piatetsky-Shapiro, G., and Smyth, P. (1996c). Knowledge discovery and data mining: Towards a unifying framework. In Proceedings of the Second International Conference on Knowledge Discovery and Data Mining (KDD-96), Portland, Oregon, USA, pages 82-88.

Gandomi, A. and Haider, M. (2015). Beyond the hype: Big data concepts, methods, and analytics. Int J. Information Management, 35(2):137-144.

Garousi, V., Felderer, M., and Mäntylä, M. V. (2016). The need for multivocal literature reviews in software engineering: complementing systematic literature reviews with grey literature. In Proceedings of the 20th International Conference on Evaluation and Assessment in Software Engineering, EASE 2016, Limerick, Ireland, June 01 - 03, 2016, pages 26:1-26:6.

Garousi, V., Felderer, M., and Mäntylä, M. V. (2019). Guidelines for including grey literature and conducting multivocal literature reviews in software engineering. Information \& Software Technology, 106:101-121.

Gertosio, C. and Dussauchoy, A. (2004). Knowledge discovery from industrial databases. J. Intelligent Manufacturing, 15(1):29-37.

Hassani, H., Huang, X., and Silva, E. (2018). Digitalisation and big data mining in banking. Big Data and Cognitive Computing, 2(3):18.

Huber, S., Wiemer, H., Schneider, D., and Ihlenfeldt, S. (2019). Dmme: Data mining methodology for engineering applications-a holistic extension to the crisp-dm model. Procedia CIRP, 79:403-408.

IBM, C. (2016). Analytics Solutions Unified Method. IBM Corporation, New Orchard Road Armonk, NY 10504.

Kitchenham, B. and Charters, S. (2007). Guidelines for performing systematic literature reviews in software engineering. EBSE Technical Report No. EBSE-2007-01.

Kitchenham, B. A., Budgen, D., and Brereton, P. (2015). Evidence-based software engineering and systematic reviews. CRC press.

Kofod-Petersen, A. (2014). How to do a structured literature review in computer science (version 0.2). Copenhagen: Alexandra Institute.

Levy, Y. and Ellis, T. J. (2006). A systems approach to conduct an effective literature review in support of information systems research. InformingSciJ, 9:181-212.

Liao, S., Chu, P., and Hsiao, P. (2012). Data mining techniques and applications - A decade review from 2000 to 2011. Expert Syst. Appl., 39(12):11303-11311.

Madni, H. A., Anwar, Z., and Shah, M. A. (2017). Data mining techniques and applications - A decade review. In 23rd International Conference on Automation and Computing, ICAC 2017, Huddersfield, United Kingdom, September 7-8, 2017, pages 1-7.

Mariani, M., Baggio, R., Fuchs, M., and Höepken, W. (2018). Business intelligence and big data in hospitality and tourism: a systematic literature review. International Journal of Contemporary Hospitality Management, 30(12):3514-3554.

Martínez-Plumed, F., Ochando, L. C., Ferri, C., Flach, P. A., Hernández-Orallo, J., Kull, M., Lachiche, N., and Ramírez-Quintana, M. J. (2017). CASP-DM: context aware standard process for data mining. CoRR, abs/1709.09003.

Morabito, V. (2016). The future of digital business innovation: Trends and practices. Springer.

Moyle, S. and Jorge, A. (2001). Ramsys-a methodology for supporting rapid remote collaborative data mining projects. In ECML/PKDD01 Workshop: Integrating Aspects of Data Mining, Decision Support and Meta-learning (IDDM-2001). 
Neto, G. T. G., Santos, W. B., Endo, P. T., and de A. Fagundes, R. A. (2019). Multivocal literature reviews in software engineering: Preliminary findings from a tertiary study. In 2019 ACM/IEEE International Symposium on Empirical Software Engineering and Measurement, ESEM 2019, Porto de Galinhas, Recife, Brazil, September 19-20, 2019, pages 1-6.

Niaksu, O. (2015). Crisp data mining methodology extension for medical domain. Baltic Journal of Modern Computing, 3(2):92.

Pyzdek, T. and Keller, P. (2003). The six sigma handbook: A complete guide for green belts, black belts, and managers at all level. New York [ua]: McGraw-Hill.

Romero, C. and Ventura, S. (2013). Data mining in education. Wiley Interdiscip. Rev. Data Min. Knowl. Discov., 3(1):12-27.

Saltz, J. S. and Shamshurin, I. (2016). Big data team process methodologies: A literature review and the identification of key factors for a project's success. In 2016 IEEE International Conference on Big Data, BigData 2016, Washington DC, USA, December 5-8, 2016, pages 2872-2879.

SAS Institute, I. (2017). SAS® Enterprise Miner ${ }^{T M}$ 14.3: Reference Help. Cary, NC: SAS Institute Inc.

Tsai, C., Lai, C., Chao, H., and Vasilakos, A. V. (2015). Big data analytics: a survey. J. Big Data, 2:21.

Vanwersch, R., Shahzad, K., Vanhaecht, K., Grefen, P., Pintelon, L., Mendling, J., Van Merode, G., and Reijers, H. (2011). Methodological support for business process redesign in health care: a literature review protocol. International Journal of Care Pathways, 15(4):119-126.

\section{PRIMARY SOURCES}

Ahangama, S. and Poo, D. C. C. (2015). What methodological attributes are essential for novice users to analytics? - an empirical study. In Human Interface and the Management of Information. Information and Knowledge in Context - 17th International Conference, HCI International 2015, Los Angeles, CA, USA, August 2-7, 2015, Proceedings, Part II, pages 77-88.

Ahmed, F., Rafique, M. Z., and Abulaish, M. (2011). A data mining framework for securing $3 \mathrm{~g}$ core network from GTP fuzzing attacks. In Information Systems Security - 7th International Conference, ICISS 2011, Kolkata, India, December 15-19, 2011, Procedings, pages 280-293.

Alazab, M., Venkatraman, S., Watters, P. A., and Alazab, M. (2011). Zero-day malware detection based on supervised learning algorithms of API call signatures. In Ninth Australasian Data Mining Conference, AusDM 2011, Ballarat, Australia, December 2011, pages 171-182.

Amani, F. and Fadlalla, A. (2017). Data mining applications in accounting: A review of the literature and organizing framework. International Journal of Accounting Information Systems, 24:32-58.

Angelov, P. (2014). Outside the box: an alternative data analytics framework. Journal of Automation Mobile Robotics and Intelligent Systems, 8(2):29-35.

Barbará, D., Couto, J., Jajodia, S., and Wu, N. (2001). ADAM: A testbed for exploring the use of data mining in intrusion detection. SIGMOD Record, 30(4):15-24.

Bashir, M. R. and Gill, A. Q. (2016). Towards an iot big data analytics framework: Smart buildings systems. In 18th IEEE International Conference on High Performance Computing and Communications; 14th IEEE International Conference on Smart City; 2nd IEEE International Conference on Data Science and Systems, HPCC/SmartCity/DSS 2016, Sydney, Australia, December 12-14, 2016, pages 1325-1332.

Behbahani, M. P., Khaddaj, S., and Choudhury, I. (2011). A multilayer data mining approach to an optimized ebusiness analytics framework. International Proceedings of Economics Development and Research, pages 66-71. 
Biliri, E., Petychakis, M., Alvertis, I., Lampathaki, F., Koussouris, S., and Askounis, D. (2014). Infusing social data analytics into future internet applications for manufacturing. In 11th IEEE/ACS International Conference on Computer Systems and Applications, AICCSA 2014, Doha, Qatar, November 10-13, 2014, pages 515-522.

Bohanec, M., Robnik-Sikonja, M., and Borstnar, M. K. (2017). Decision-making framework with doubleloop learning through interpretable black-box machine learning models. Industrial Management and Data Systems, 117(7):1389-1406.

Brisson, L. and Collard, M. (2008). How to semantically enhance a data mining process? In Enterprise Information Systems, 10th International Conference, ICEIS 2008, Barcelona, Spain, June 12-16, 2008, Revised Selected Papers, pages 103-116.

Büchner, A. G. and Mulvenna, M. D. (1998). Discovering internet marketing intelligence through online analytical web usage mining. SIGMOD Record, 27(4):54-61.

Cannataro, M. and Comito, C. (2003). A data mining ontology for grid programming. In Proc. 1st Int. Workshop on Semantics in Peer-to-Peer and Grid Computing, pages 113-134. Citeseer.

Cao, L. (2010). Domain-driven data mining: Challenges and prospects. IEEE Trans. Knowl. Data Eng., 22(6):755-769.

Cao, L., Schurmann, R., and Zhang, C. (2005). Domain-driven in-depth pattern discovery: a practical methodology. In Australian Data Mining Conference. The University of Technology, Sydney.

Cao, L. and Zhang, C. (2007). The evolution of KDD: towards domain-driven data mining. IJPRAI, 21(4):677-692.

Cao, L. and Zhang, C. (2008). Domain driven data mining. In Data Mining and Knowledge Discovery Technologies, pages 196-223. IGI Global.

Capozzoli, A., Piscitelli, M. S., Gorrino, A., Ballarini, I., and Corrado, V. (2017). Data analytics for occupancy pattern learning to reduce the energy consumption of hvac systems in office buildings. Sustainable cities and society, 35:191-208.

Chatzikonstantinou, G., Kontogiannis, K., and Attarian, I. (2013). A goal driven framework for software project data analytics. In Advanced Information Systems Engineering - 25th International Conference, CAiSE 2013, Valencia, Spain, June 17-21, 2013. Proceedings, pages 546-561.

Chee, T. C., Baharudin, A. S., and Karkonasasi, K. (2016). Data mining framework for test time optimization in industrial electronics manufacturing enterprise. International Journal of Applied Engineering Research.

Chen, H., Kazman, R., and Haziyev, S. (2016). Agile big data analytics development: An architecturecentric approach. In 49th Hawaii International Conference on System Sciences, HICSS 2016, Koloa, HI, USA, January 5-8, 2016, pages 5378-5387.

Chen, Y., Gao, W., Wang, Z., Miao, J., and Jiang, D. (2001). Mining audio/visual database for speech driven face animation. In Proceedings of the IEEE International Conference on Systems, Man \& Cybernetics: "e-Systems and e-Man for Cybernetics in Cyberspace", Tucson, Arizona, USA, 7-10 October 2001, pages 2638-2643.

Chernov, S., Chernogorov, F., Petrov, D., and Ristaniemi, T. (2014). Data mining framework for random access failure detection in LTE networks. In 25th IEEE Annual International Symposium on Personal, Indoor, and Mobile Radio Communication, PIMRC 2014, Washington DC, USA, September 2-5, 2014, pages 1321-1326.

Chernov, S., Petrov, D., and Ristaniemi, T. (2015). Location accuracy impact on cell outage detection in LTE-A networks. In International Wireless Communications and Mobile Computing Conference, IWCMC 2015, Dubrovnik, Croatia, August 24-28, 2015, pages 1162-1167. 
Chien, C., Diaz, A. C., and Lan, Y. (2014). A data mining approach for analyzing semiconductor MES and FDC data to enhance overall usage effectiveness (OUE). Int. J. Comput. Intell. Syst., 7(sup2):52-65.

Çinicioğlu, E. N., Ertek, G., Demirer, D., and Yörük, H. E. (2011). A framework for automated association mining over multiple databases. In 2011 International Symposium on Innovations in Intelligent Systems and Applications, pages 79-85. IEEE.

Cuzzocrea, A., Psaila, G., and Toccu, M. (2016). An innovative framework for effectively and efficiently supporting big data analytics over geo-located mobile social media. In Proceedings of the 20th International Database Engineering \& Applications Symposium, IDEAS 2016, Montreal, QC, Canada, July 11-13, 2016, pages 62-69.

Debuse, J. (2007). Extending data mining methodologies to encompass organizational factors. Systems Research and Behavioral Science: The Official Journal of the International Federation for Systems Research, 24(2):183-190.

Deng, J. D., Purvis, M. K., and Purvis, M. (2011). Software effort estimation: Harmonizing algorithms and domain knowledge in an integrated data mining approach. IJIIT, 7(3):41-53.

Deng, X., Ghanem, M., and Guo, Y. (2009). Real-time data mining methodology and a supporting framework. In Third International Conference on Network and System Security, NSS 2009, Gold Coast, Queensland, Australia, October 19-21, 2009, pages 522-527.

Ding, Q. and Daniel, C. (2007). Multimedia data mining framework for banner images. In Multimedia Data Mining and Knowledge Discovery, pages 448-457. Springer.

Doreswamy (2008). A survey for data mining frame work for polymer matrix composite engineering materials design applications. Int. J. Comput. Intell. Syst., 1(4):313-328.

Du, M., Li, F., Zheng, G., and Srikumar, V. (2017). Deeplog: Anomaly detection and diagnosis from system logs through deep learning. In Proceedings of the 2017 ACM SIGSAC Conference on Computer and Communications Security, CCS 2017, Dallas, TX, USA, October 30 - November 03, 2017, pages $1285-1298$.

Ertek, G., Chi, X., and Zhang, A. N. (2017). A framework for mining RFID data from schedule-based systems. IEEE Trans. Systems, Man, and Cybernetics: Systems, 47(11):2967-2984.

Fan, Y., Ye, Y., and Chen, L. (2016). Malicious sequential pattern mining for automatic malware detection. Expert Syst. Appl., 52:16-25.

François, D. (2008). Methodology and standards for data analysis with machine learning tools. In ESANN 2008, 16th European Symposium on Artificial Neural Networks, Bruges, Belgium, April 23-25, 2008, Proceedings, pages 239-246.

Ganesh, M., Han, E., Kumar, V., Shekhar, S., and Srivastava, J. (1996). Visual data mining: Framework and algorithm development. Department of Computing and Information Sciences, University of Minnesota, MN, USA.

García, D., Creus, R., Minoves, M., Pardo, X., Quevedo, J., and Puig, V. (2017). Data analytics methodology for monitoring quality sensors and events in the barcelona drinking water network. Journal of Hydroinformatics, 19(1):123-137.

Gomes, J. B., Phua, C., and Krishnaswamy, S. (2013). Where will you go? mobile data mining for next place prediction. In Data Warehousing and Knowledge Discovery - 15th International Conference, DaWaK 2013, Prague, Czech Republic, August 26-29, 2013. Proceedings, pages 146-158.

Guan, Q. and Fu, S. (2010). auto-aid: A data mining framework for autonomic anomaly identification in networked computer systems. In 29th International Performance Computing and Communications Conference, IPCCC 2010, 9-11 December 2010, Albuquerque, NM, USA, pages 73-80. 
Güder, M., Salor-Durna, Ö., Çadirci, I., Ozkan, B., and Altintas, E. (2014). Data mining framework for power quality event characterization of iron and steel plants. In 2014 IEEE Industry Application Society Annual Meeting, Vancouver, BC, Canada, October 5-9, 2014, pages 1-11.

Hang, Y. and Fong, S. (2009). A framework of business intelligence-driven data mining for e-business. In International Conference on Networked Computing and Advanced Information Management, NCM 2009, Fifth International Joint Conference on INC, IMS and IDC: INC 2009: International Conference on Networked Computing, IMS 2009: International Conference on Advanced Information Management and Service, IDC 2009: International Conference on Digital Content, Multimedia Technology and its Applications, Seoul, Korea, August 25-27, 2009, pages 1964-1970.

Haruechaiyasak, C., Shyu, M., and Chen, S. (2004). A data mining framework for building A web-page recommender system. In Proceedings of the 2004 IEEE International Conference on Information Reuse and Integration, IRI - 2004, November 8-10, 2004, Las Vegas Hilton, Las Vegas, NV, USA, pages 357-362.

Hossain, M., Bridges, S. M., and Jr., R. B. V. (2003). Adaptive intrusion detection with data mining. In Proceedings of the IEEE International Conference on Systems, Man \& Cybernetics: Washington, D.C., USA, 5-8 October 2003, pages 3097-3103.

Hu, X., Xu, P., Wu, S., Asgari, S., and Bergsneider, M. (2010). A data mining framework for time series estimation. Journal of Biomedical Informatics, 43(2):190-199.

Huang, X., Chen, S., Shyu, M., and Zhang, C. (2002). Mining high-level user concepts with multiple instance learning and relevance feedback for content-based image retrieval. In Mining Multimedia and Complex Data, KDD Workshop MDM/KDD 2002, PAKDD Workshop KDMCD 2002, Revised Papers, pages 50-67.

Kabir, M. H. (2016). Data mining framework for generating sales decision making information using association rules. International Journal of Advanced Computer Science and Applications, 7(5):378-385.

Kamrani, A., Rong, W., and Gonzalez, R. (2001). A genetic algorithm methodology for data mining and intelligent knowledge acquisition. Computers \& Industrial Engineering, 40(4):361-377.

Kang, S., Kim, E., Shim, J., Cho, S., Chang, W., and Kim, J. (2017). Mining the relationship between production and customer service data for failure analysis of industrial products. Computers \& Industrial Engineering, 106:137-146.

Karimi-Majd, A. and Mahootchi, M. (2015). A new data mining methodology for generating new service ideas. Inf. Syst. E-Business Management, 13(3):421-443.

Khan, D. M., Mohamudally, N., and Babajee, D. K. R. (2013). A unified theoretical framework for data mining. In Proceedings of the First International Conference on Information Technology and Quantitative Management, ITQM 2013, Dushu Lake Hotel, Sushou, China, 16-18 May, 2013, pages 104-113.

Kiayias, A., Neumann, J., Walluck, D., and McCusker, O. (2009). A combined fusion and data mining framework for the detection of botnets. In 2009 Cybersecurity Applications \& Technology Conference for Homeland Security, pages 273-284. IEEE.

Kisilevich, S., Keim, D. A., and Rokach, L. (2013). A gis-based decision support system for hotel room rate estimation and temporal price prediction: The hotel brokers' context. Decision Support Systems, 54(2):1119-1133.

Kühn, A., Joppen, R., Reinhart, F., Röltgen, D., von Enzberg, S., and Dumitrescu, R. (2018). Analytics canvas-a framework for the design and specification of data analytics projects. Procedia CIRP, 70:162-167.

Kumar, A., Shankar, R., Choudhary, A., and Thakur, L. S. (2016). A big data mapreduce framework for fault diagnosis in cloud-based manufacturing. International Journal of Production Research, 54(23):7060-7073. 
Kumar, A., Shankar, R., and Thakur, L. S. (2018). A big data driven sustainable manufacturing framework for condition-based maintenance prediction. J. Comput. Science, 27:428-439.

Kurgan, L. A. and Musílek, P. (2006). A survey of knowledge discovery and data mining process models. Knowledge Engineering Review, 21(1):1-24.

Lau, R. Y. K., Zhang, W., and Xu, W. (2018). Parallel aspect-oriented sentiment analysis for sales forecasting with big data. Production and Operations Management, 27(10):1775-1794.

Lawler, J. and Joseph, A. (2017). Big data analytics methodology in the financial industry. Information Systems Education Journal, 15(4):38.

Lee, W., Nimbalkar, R. A., Yee, K. K., Patil, S. B., Desai, P. H., Tran, T. T., and Stolfo, S. J. (2000). A data mining and CIDF based approach for detecting novel and distributed intrusions. In Recent Advances in Intrusion Detection, Third International Workshop, RAID 2000, Toulouse, France, October 2-4, 2000, Proceedings, pages 49-65.

Lee, W., Stolfo, S. J., Chan, P. K., Eskin, E., Fan, W., Miller, M., Hershkop, S., and Zhang, J. (2001). Real time data mining-based intrusion detection. In Proceedings DARPA Information Survivability Conference and Exposition II. DISCEX'01, volume 1, pages 89-100. IEEE.

Lee, W., Stolfo, S. J., and Mok, K. W. (1999). Mining in a data-flow environment: Experience in network intrusion detection. In Proceedings of the Fifth ACM SIGKDD International Conference on Knowledge Discovery and Data Mining, San Diego, CA, USA, August 15-18, 1999, pages 114-124.

Lemieux, V. L. (2016). Innovating good regulatory practice using mixed-initiative social media analytics and visualization. In 2016 Conference for E-Democracy and Open Government, CeDEM 2016, Krems, Austria, May 18-20, 2016, pages 207-212.

Lenz, J., Wuest, T., and Westkämper, E. (2018). Holistic approach to machine tool data analytics. Journal of manufacturing systems, 48:180-191.

Létourneau, S., Yang, C., Drummond, C., Scarlett, E., Valdés, J., and Zaluski, M. (2005). A domain independent data mining methodology for prognostics. In Essential technologies for successful prognostics: proceedings of the 59th Meeting of the Society for Machinery Failure Prevention Technology, Virginia Beach, Virginia, April 18-21, 2005.

Liu, F., Xu, R., Fan, W., and Jiang, Z. (2018). Data analytics approach for train timetable performance measures using automatic train supervision data. IET Intelligent Transport Systems, 12(7):568-577.

Lu, Q., Lyu, Z.-J., Xiang, Q., Zhou, Y., and Bao, J. (2017). Research on data mining service and its application case in complex industrial process. In 2017 13th IEEE Conference on Automation Science and Engineering (CASE), pages 1124-1129. IEEE.

Luna, J. M., Castro, C., and Romero, C. (2017). MDM tool: A data mining framework integrated into moodle. Comp. Applic. in Engineering Education, 25(1):90-102.

Mahmood, A., Shi, K., Khatoon, S., and Xiao, M. (2013). Data mining techniques for wireless sensor networks: A survey. IJDSN, 9.

Marbán, O., Mariscal, G., Ruiz, E. M., and Segovia, J. (2007). An engineering approach to data mining projects. In Intelligent Data Engineering and Automated Learning - IDEAL 2007, 8th International Conference, Birmingham, UK, December 16-19, 2007, Proceedings, pages 578-588.

Marban, O., Mariscal, G., and Segovia, J. (2009). A data mining and knowledge discovery process model. Data Mining and Knowledge Discovery in Real Life Applications, edited by P. Julio and K. Adem, Paris, I-Tech, Vienna, Austria, pages 438-453.

Marbán, O., Segovia, J., Menasalvas, E., and Fernández-Baizán, C. (2009). Toward data mining engineering: A software engineering approach. Information systems, 34(1):87-107. 
Mariscal, G., Marbán, Ó., and Fernández, C. (2010). A survey of data mining and knowledge discovery process models and methodologies. Knowledge Eng. Review, 25(2):137-166.

Mobasher, B. (2007). Data mining for web personalization. In The Adaptive Web, Methods and Strategies of Web Personalization, pages 90-135.

Murnion, P. and Helfert, M. (2011). A framework for decision support for learning management systems. In 10th European Conference on e-Learning ECEL-2011. Brighton, UK.

Nestorov, S. and Jukic, N. (2003). Ad-hoc association-rule mining within the data warehouse. In 36th Hawaii International Conference on System Sciences (HICSS-36 2003), CD-ROM / Abstracts Proceedings, January 6-9, 2003, Big Island, HI, USA, page 232.

Niesen, T., Houy, C., Fettke, P., and Loos, P. (2016). Towards an integrative big data analysis framework for data-driven risk management in industry 4.0. In 49th Hawaii International Conference on System Sciences, HICSS 2016, Koloa, HI, USA, January 5-8, 2016, pages 5065-5074.

Nohuddin, P., Zainol, Z., Lee, A. S. H., Nordin, I., and Yusoff, Z. (2018). A case study in knowledge acquisition for logistic cargo distribution data mining framework. International Journal of Advanced and Applied Sciences, 5(1):8-14.

Ortega, J. P., Iturbide, E., Olivares, V., Hidalgo, M. A., Almanza, N., and Rebollar, A. M. (2015). A data preparation methodology in data mining applied to mortality population databases. In New Contributions in Information Systems and Technologies - Volume 1 [WorldCIST'15, Azores, Portugal, April 1-3, 2015]., pages 1173-1182.

Osman, A. M. S., Elragal, A., and Bergvall-Kåreborn, B. (2017). Big data analytics and smart cities: A loose or tight couple? In 10th International Conference on Connected Smart Cities 2017 (CSC 2017), Lisbon, 20-22 July 2017, pages 157-168. IADIS.

Park, G., Chung, L., Zhao, L., and Supakkul, S. (2017). A goal-oriented big data analytics framework for aligning with business. In Third IEEE International Conference on Big Data Computing Service and Applications, BigDataService 2017, Redwood City, CA, USA, April 6-9, 2017, pages 31-40.

Pournaras, E., Nikolic, J., Velásquez, P., Trovati, M., Bessis, N., and Helbing, D. (2016). Self-regulatory information sharing in participatory social sensing. EPJ Data Sci., 5(1):14.

Pouyanfar, S. and Chen, S. (2016). Semantic concept detection using weighted discretization multiple correspondence analysis for disaster information management. In 17th IEEE International Conference on Information Reuse and Integration, IRI 2016, Pittsburgh, PA, USA, July 28-30, 2016, pages 556-564.

Puthal, D., Nepal, S., Ranjan, R., and Chen, J. (2016). A secure big data stream analytics framework for disaster management on the cloud. In 18th IEEE International Conference on High Performance Computing and Communications; 14th IEEE International Conference on Smart City; 2nd IEEE International Conference on Data Science and Systems, HPCC/SmartCity/DSS 2016, Sydney, Australia, December 12-14, 2016, pages 1218-1225.

Rahman, F. A., Desa, M. I., and Wibowo, A. (2011). A review of kdd-data mining framework and its application in logistics and transportation. In The 7th International Conference on Networked Computing and Advanced Information Management, pages 175-180. IEEE.

Rendall, R., Lu, B., Castillo, I., Chin, S.-T., Chiang, L. H., and Reis, M. S. (2017). A unifying and integrated framework for feature oriented analysis of batch processes. Industrial \& Engineering Chemistry Research, 56(30):8590-8605.

Reutterer, T., Hornik, K., March, N., and Gruber, K. (2017). A data mining framework for targeted category promotions. Journal of Business Economics, 87(3):337-358.

Segarra, L. L., Almalki, H., Elabd, J., Gonzalez, J., Marczewski, M., Alrasheed, M., and Rabelo, L. (2016). A framework for boosting revenue incorporating big data. Journal of Innovation Management, 4(1):39-68. 
Shahbaz, M., Masood, S. A., Shaheen, M., and Khan, A. (2010). Data mining methodology in perspective of manufacturing databases. J Am Sci.

Shao, Z., Liu, J., and Zhu, X. (2008). Image mining for generating ontology databases of geographical entities. Proceedings of the 8th International Symposium on Spatial Accuracy Assessment in Natural Resources and Environmental Sciences.

Sharma, S. and Osei-Bryson, K. (2008). Organization-ontology based framework for implementing the business understanding phase of data mining projects. In 41st Hawaii International International Conference on Systems Science (HICSS-41 2008), Proceedings, 7-10 January 2008, Waikoloa, Big Island, HI, USA, page 77.

Sharma, S. and Osei-Bryson, K. (2009). Framework for formal implementation of the business understanding phase of data mining projects. Expert Syst. Appl., 36(2):4114-4124.

Shin, M. S. and Jeong, K. J. (2005). An alert data mining framework for network-based intrusion detection system. In Information Security Applications, 6th International Workshop, WISA 2005, Jeju Island, Korea, August 22-24, 2005, Revised Selected Papers, pages 38-53.

Shrivastava, S. and Pal, S. N. (2017). A big data analytics framework for enterprise service ecosystems in an e-governance scenario. In Proceedings of the 10th International Conference on Theory and Practice of Electronic Governance, ICEGOV 2017, New Delhi, India, March 07 - 09, 2017, pages 5-11.

Simoff, S. J. and Galloway, J. (2008). Visual discovery of network patterns of interaction between attributes. In Visual Data Mining - Theory, Techniques and Tools for Visual Analytics, pages 172-195. Springer.

Singh, K., Guntuku, S. C., Thakur, A., and Hota, C. (2014). Big data analytics framework for peer-to-peer botnet detection using random forests. Inf. Sci., 278:488-497.

Singh, S., Prasad, A., Srivastava, K., and Bhattacharya, S. (2016). A cellular logic array based data mining framework for object detection in video surveillance system. In 2016 2nd International Conference on Next Generation Computing Technologies (NGCT), pages 719-724. IEEE.

Singh, S., Vajirkar, P., and Lee, Y. (2003). Context-based data mining using ontologies. In Conceptual Modeling - ER 2003, 22nd International Conference on Conceptual Modeling, Chicago, IL, USA, October 13-16, 2003, Proceedings, pages 405-418.

Smith, K. A., Willis, R. J., and Brooks, M. (2000). An analysis of customer retention and insurance claim patterns using data mining: A case study. Journal of the operational research society, 51(5):532-541.

Solarte, J. (2002). A Proposed Data Mining Methodology and its Application to Industrial Engineering. $\mathrm{PhD}$ thesis, University of Tennessee.

Strohbach, M., Ziekow, H., Gazis, V., and Akiva, N. (2015). Towards a big data analytics framework for iot and smart city applications. In Modeling and processing for next-generation big-data technologies, pages 257-282. Springer.

Sun, J., Xu, W., Ma, J., and Sun, J. (2015). Leverage raf to find domain experts on research social network services: A big data analytics methodology with mapreduce framework. International Journal of Production Economics, 165:185-193.

Tavares, R., Vieira, R., and Pedro, L. (2017). A preliminary proposal of a conceptual educational data mining framework for science education: Scientific competences development and self-regulated learning. In 2017 International Symposium on Computers in Education (SIIE), pages 1-6. IEEE.

Torres, P., Marques, P., Marques, H., Dionisio, R., Alves, T., Pereira, L., and Ribeiro, J. (2017). Data analytics for forecasting cell congestion on LTE networks. In Network Traffic Measurement and Analysis Conference, TMA 2017, Dublin, Ireland, June 21-23, 2017, pages 1-6. 
van Rooyen, M. and Simoff, S. J. (2008). A strategic analytics methodology. In ICSOFT 2008 Proceedings of the Third International Conference on Software and Data Technologies, Volume ISDM/ABF, Porto, Portugal, July 5-8, 2008, pages 20-28.

Wang, L. (2015). Data mining in functional test content optimization. In The 20th Asia and South Pacific Design Automation Conference, ASP-DAC 2015, Chiba, Japan, January 19-22, 2015, pages 308-315.

Wang, L. (2017). Experience of data analytics in EDA and test - principles, promises, and challenges. IEEE Trans. on CAD of Integrated Circuits and Systems, 36(6):885-898.

Wijayasekara, D., Linda, O., and Manic, M. (2011). CAVE-SOM: immersive visual data mining using 3d self-organizing maps. In The 2011 International Joint Conference on Neural Networks, IJCNN 2011, San Jose, California, USA, July 31 - August 5, 2011, pages 2471-2478.

Xiang, L. (2009a). Context-aware data mining methodology for supply chain finance cooperative systems. In Fifth International Conference on Autonomic and Autonomous Systems, ICAS 2009, Valencia, Spain, 20-25 April 2009, pages 301-306.

Xiang, L. (2009b). Integrating context-aware and fuzzy rule to data mining model for supply chain finance cooperative systems. In The Fourth International Conference on Software Engineering Advances, ICSEA 2009, 20-25 September 2009, Porto, Portugal, pages 471-476.

$\mathrm{Xu}, \mathrm{S}$. and Qiu, M. (2008). A privacy preserved data mining framework for customer relationship management. Journal of Relationship Marketing, 7(3):309-322.

Yang, L. and Shi, Z. (2010). An efficient data mining framework on hadoop using java persistence API. In 10th IEEE International Conference on Computer and Information Technology, CIT 2010, Bradford, West Yorkshire, UK, June 29-July 1, 2010, pages 203-209.

Yang, Y., Zheng, Z., Huang, C., Li, K., and Dai, H. (2016). A novel hybrid data mining framework for credit evaluation. In Collaborate Computing: Networking, Applications and Worksharing - 12th International Conference, CollaborateCom 2016, Beijing, China, November 10-11, 2016, Proceedings, pages 16-26.

Yi, W., Teng, F., and Xu, J. (2016). Noval stream data mining framework under the background of big data. Cybernetics and Information Technologies, 16(5):69-77.

Ying, Y., Yinghong, W., Rong, J., and Liquan, J. (2014). Domain driven data mining for customer demand discovery. In 2014 11th International Conference on Service Systems and Service Management (ICSSSM), pages 1-6. IEEE.

Yu, G., Köppen, M., Chen, S., and Niu, X., editors (2009). 9th International Conference on Hybrid Intelligent Systems (HIS 2009), August 12-14, 2009, Shenyang, China. IEEE Computer Society.

Yu, Z., Fung, B. C., and Haghighat, F. (2013). Extracting knowledge from building-related data-a data mining framework. In Building Simulation, volume 6(2), pages 207-222. Springer.

Yuan, B. and Herbert, J. (2014). A cloud-based mobile data analytics framework: Case study of activity recognition using smartphone. In 2nd IEEE International Conference on Mobile Cloud Computing, Services, and Engineering, MobileCloud 2014, Oxford, United Kingdom, April 8-11, 2014, pages 220-227.

Yuan, B., Herbert, J., and Emamian, Y. (2014). Smartphone-based activity recognition using hybrid classifier - utilizing cloud infrastructure for data analysis. In PECCS 2014 - Proceedings of the 4th International Conference on Pervasive and Embedded Computing and Communication Systems, Lisbon, Portugal, 7-9 January, 2014, pages 14-23.

Yun, Z., Weihua, L., and Yang, C. (2014). Applying balanced scordcard strategic performance management to crisp-dm. In 2014 International Conference on Information Science, Electronics and Electrical Engineering, volume 3, pages 2009-2014. IEEE. 
Zaghloul, M. M., Ali-Eldin, A., and Salem, M. (2013). Towards a self-service data analytics framework. International Journal of Computer Applications, 80(9).

Zaki, M. and Sobh, T. S. (2005). NCDS: data mining for discovering interesting network characteristics. Information \& Software Technology, 47(3):189-198.

Zaluski, M., Létourneau, S., Bird, J., and Yang, C. (2011). Developing data mining-based prognostic models for cf-18 aircraft. Journal of Engineering for Gas Turbines and Power, 133(10):101601.

Zhang, W., Lau, R. Y. K., and Li, C. (2014). Adaptive big data analytics for deceptive review detection in online social media. In Proceedings of the International Conference on Information Systems - Building a Better World through Information Systems, ICIS 2014, Auckland, New Zealand, December 14-17, 2014.

Zhao, K., Liu, B., Tirpak, T. M., and Xiao, W. (2005). Opportunity map: a visualization framework for fast identification of actionable knowledge. In Proceedings of the 2005 ACM CIKM International Conference on Information and Knowledge Management, Bremen, Germany, October 31 - November 5 , 2005 , pages 60-67.

Zhong, R. Y., Xu, C., Chen, C., and Huang, G. Q. (2017). Big data analytics for physical internet-based intelligent manufacturing shop floors. International journal of production research, 55(9):2610-2621. 